\title{
Spectral sequences for cyclic homology
}

\author{
D. Kaledin* \\ To Maxim Kontsevich, for his 50th birthday.
}

\section{Contents}

1 Co-periodic cyclic homology. 4

1.1 Mixed complexes. . . . . . . . . . . . . . . . . 4

1.2 Small categories. . . . . . . . . . . . . . 5

1.3 Cyclic homology. . . . . . . . . . . . . . 8

2 Conjugate spectral sequence. $\quad \mathbf{1 0}$

2.1 Filtrations. . . . . . . . . . . . . . . . . . 10

2.2 Edgewise subdivision. . . . . . . . . . . . . . . . . . . . 13

2.3 Localized conjugate filtration. . . . . . . . . . . . . . . 16

2.4 DG algebras. . . . . . . . . . . . . . . 17

3 Tate cohomology.

3.1 Relative Tate cohomology. . . . . . . . . . . . . . . . . 19

3.2 Conjugate filtration. . . . . . . . . . . . . . . 22

3.3 Splitting for DG algebras. . . . . . . . . . . . . 24

4 Characteristic 2. 26

4.1 Trace functors. . . . . . . . . . . . . . . 27

4.2 Quotients of the conjugate filtration. . . . . . . . 29

5 Degeneration. 32

5.1 Conjugate degeneration. . . . . . . . . . . . . . . . . . 32

5.2 Hodge-to de Rham degeneration. . . . . . . . . . . . 36

*Partially supported by RSF, grant 14-50-00005, and the Dynasty Foundation award 


\section{Introduction.}

In modern non-commutative algebraic geometry as formalized for example by M. Kontsevich and Y. Soibelman [KS], algebraic varieties are replaced by associative unital DG algebras $A$. considered up to a derived Morita equivalence. The role of differential forms is played by Hochschild homology classes of $A_{\text {. }}$, and de Rham cohomology corresponds to periodic cyclic homology $H P .(A$.$) . Periodic cyclic homology is related to Hochschild ho-$ mology by a standard spectral sequence. If $A$. is derived Morita-equivalent to a smooth algebraic variety $X$, then the spectral sequence reduces to the classical Hodge-to-de Rham spectral sequence of P. Deligne. Because of this, one also calls it the Hodge-to-de Rham spectral sequence in the general case.

If the smooth variety $X$ is also proper, and the base ring is a field of characteristic 0 , then the Hodge-to-de Rham spectral sequence degenerates. This is Deligne's reformulation of the classical Hodge theory, and there is also an alternative purely algebraic proof due to Deligne and L. Illusie [DI].

Motivated by this, Kontsevich and Soibelman conjectured in [KS] that if a general DG algebra $A$. over a field of characteristic 0 is homologically smooth and homologically proper, then the Hodge-to-de Rham spectral sequence degenerates.

This conjecture has been largely proved in Ka2, by an adaptation of the method of Deligne and Illusie. However, the argument in that paper suffers from two related drawbacks:

- One has to impose an additional assumption that the DG algebra $A$. is question is concentrated in non-negative homological degrees (that is, $A_{i}=0$ for $\left.i>0\right)$.

- To pass form algebras to DG algebras, one uses Dold-Kan equivalence and simplicial methods. This is what forces one to impose the assumption above, and this is what makes a large part of the argument very hard to understand.

In this paper, we revisit the subject, and we give a proof of KontsevichSoibelman Degeneration Conjecture free from any additional technical assumptions.

Our method is still the same - it is based on reduction to positive characteristic and adapting the approach of [DI]. In principle, one could remove the assumption (•) by using simplicial-cosimplicial objects as e.g. in [Ka3]. However, this would make the argument even more opaque. Instead, we 
opt for an earlier approach tried in the unpublished preprint [Ka1. This is much closer to [DI], in that it uses explicitly two different spectral sequences that exist in positive characteristic - the Hodge-to-de Rham spectral sequence, on one hand, and the so-called conjugate spectral sequence on the other hand. The spectral sequences are completely different, but they have the same first page and the same last page. So, what one actually proves is that the conjugate spectral sequence degenerates, under some assumptions; the Hodge-to-de Rham sequence then degenerates for dimension reasons.

For associative algebras of finite homological dimension, it is relatively easy to construct a non-commutative version of the conjugate spectral sequence, and this has been essentially done in Ka1]. However, for general DG algebras, the question is much more delicate. It took a while to realize that the sequence in question simply does not exist — or rather, it does exist, but does not converge to periodic cyclic homology. What it converges to is a completely new additive invariant of DG algebras and DG categories constructed in [Ka5] under the name of co-periodic cyclic homology and denoted $\overline{H P}$. $(A$.$) . In retrospect, this state of affairs has been also suggested$ by Kontsevich 10 years ago Ko1, Ko2, but the major push for actually developing the theory has been given by recent works of $\mathrm{A}$. Beilinson $\mathrm{Be}$ and $\mathrm{B}$. Bhatt $[\mathrm{Bh}$. Whatever the origins of the theory are, now we know that co-periodic cyclic homology does exist, it has a conjugate spectral sequence converging to it, and for a homologically smooth and homologically bounded DG algebra $A$., there is a comparison theorem providing a canonical isomorphism $H P_{.}\left(A_{\bullet}\right) \cong \overline{H P}$. $\left(A_{\text {. }}\right)$. Therefore a Deligne-Illusie type of argument for degeneration should still be possible. This is what the present paper provides.

A couple of words about the organization of the paper. Out of necessity, a large part of it is a recapitulation of my earlier papers. In particular, Section 1 and Section 2 contain the relevant results of Ka5. Section 1 is concerned with general results about co-periodic cyclic homology, summarized in Theorem 1.3, and Section 2 contains the construction of the conjugate spectral sequence. Section 3 starts with some general results on Tate (co)homology of finite groups. Everything is completely standard but we do not know any good references (there are some intersections with Ka6, Subsection 6.3]). Then we apply the results to define a relative version of Tate cohomology, and use it to prove results about co-periodic cyclic homology. In particular, this includes a degeneration criterion (Proposition 3.6). At this point, we can already prove all out degeneration results; however, we make a detour and use the opportunity to correct one fault of [Ka5] - 
namely, we construct the conjugate spectral sequence in characteristic 2, the case excluded in [Ka5] for reasons explained in [Ka5, Subsection 5.5]. This is the subject of Section 4. The technology used is a combination of some splitting results of [Ka6, Section 6] and the notion of a trace functor of Ka3]. Finally, in Section 5, we prove our degeneration results. This includes Theorem 5.4 equivalent to the Degeneration Conjecture of [KS].

Acknowledgement. For several reasons at once, it is a great pleasure to dedicate this paper to Maxim Kontsevich. It was him who posed the original problem. He then actively encouraged my earlier work on it, and at the same time, was consistently unhappy about the unnecessary technical complications of [Ka2]. In retrospect, it was also him who showed a better way to approach the subject, although at the time, I didn't quite understand what he meant. Another mathematician to whom this paper owes a lot is Vadim Vologodsky. In particular, he always insisted that the notion of a conjugate spectral sequence omitted in Ka2 should be an integral part of the story. Moreover, a crucial technical idea that appears in [Ka6, Section 6] is also due to him. I am also very grateful to David Kazhdan and Vladimir Hinich for their interest in this work, and for encouraging me to try to settle the question once and for all. Finally, in the years that passed since Ka2], I had an opportunity to discuss the subject with many people; I want to specifically mention very useful conversations with Alexandre Beilinson, Bhargav Bhatt, Boris Feigin, and Boris Tsygan.

\section{Co-periodic cyclic homology.}

In this section, we recall main facts about co-periodic cyclic homology introduced in [Ka5, together with some terminology and notation.

1.1 Mixed complexes. A mixed complex $\left\langle V_{.}, B\right\rangle$ in an abelian category $\mathcal{E}$ is a complex $V$. in $\mathcal{E}$ equipped with a map of complexes $B: V_{\bullet} \rightarrow V_{\bullet}[-1]$ such that $B^{2}=0$ (we will drop $B$ from notaton when it is clear from the context). The periodic expansion $\operatorname{Per}\left(V_{\bullet}\right)$ of a mixed complex $V_{\text {. is the }}$ complex

$$
\operatorname{Per}\left(V_{\bullet}\right)=V_{\bullet}((u))
$$

with the differential $d+B u$, where $d$ is the differential in the complex $V$, $u$ is a formal generator of cohomological degree 2 , and $V .((u))$ is shorthand for "formal Laurent power series in $u$ with coefficients in $V$.". Analogously, 
co-periodic and polynomial periodic expansions $\overline{\operatorname{Per}}\left(V_{\bullet}\right), \operatorname{per}\left(V_{\bullet}\right)$ are given by

$$
\overline{\operatorname{Per}}\left(V_{\bullet}\right)=V_{\bullet}\left(\left(u^{-1}\right)\right), \quad \operatorname{per}\left(V_{\bullet}\right)=V_{\bullet}\left[u, u^{-1}\right],
$$

again with the differential $d+u B$. By definition, the space of Taylor power series $V_{\bullet}[[u]] \subset V_{\bullet}((u))$ is a subcomplex in the periodic expansion $\operatorname{Per}\left(V_{\bullet}\right)$; the expansion $\operatorname{Exp}\left(V_{\bullet}\right)$ is the quotient complex

$$
\operatorname{Exp}\left(V_{\bullet}\right)=V_{\bullet}\left[u^{-1}\right]=V_{\bullet}((u)) / u V \cdot[[u]] .
$$

Multiplication by $u$ induces an invertible degree- 2 endomorphism of the complexes $\operatorname{Per}\left(V_{\bullet}\right), \overline{\operatorname{Per}}\left(V_{\bullet}\right), \operatorname{per}\left(V_{\bullet}\right)$ and a non-invertible periodicity map $u: \operatorname{Exp}\left(V_{\bullet}\right) \rightarrow \operatorname{Exp}\left(V_{\bullet}\right)[2]$. We have

$$
\operatorname{Per}\left(V_{\bullet}\right) \cong \lim _{\leftarrow}^{\leftarrow} \underset{\operatorname{Exp}}{\leftarrow}\left(V_{\bullet}\right)
$$

Since a Laurent polynomial in $u$ is a Laurent power series both in $u$ and in $u^{-1}$, we have natural functorial maps

$$
\operatorname{Per}\left(V_{\bullet}\right) \longleftarrow \operatorname{per}\left(V_{\bullet}\right) \longrightarrow \overline{\operatorname{Per}}\left(V_{\bullet}\right)
$$

for any mixed complex $V$. If $V$. is concentrated in a finite range of degrees, then both maps are isomorphisms, but in general, they are not.

Example 1.1. Assume given a module $E$ over a ring $R$, and assume that a cyclic group $\mathbb{Z} / n \mathbb{Z}$ of some order $n$ acts on $E$, with $\sigma: E \rightarrow E$ being the generator. Then the length-2 complex

$$
E \stackrel{\text { id }-\sigma}{\longrightarrow} E
$$

has a natural structure of a mixed complex, with the map $B$ given by

$$
B=\mathrm{id}+\sigma+\cdots+\sigma^{n-1}: E_{\sigma} \rightarrow E^{\sigma} .
$$

The expansion of the complex (1.3) is the standard homology complex $C .(\mathbb{Z} / n \mathbb{Z}, E)$, and the periodic expansion is the standard Tate homology complex $\check{C} .(\mathbb{Z} / n \mathbb{Z}, E)$.

1.2 Small categories. In a sense, the standard example of a mixed complex that appears in nature combines the complexes of Example 1.1 for all integers $n \geq 1$. To package the data, it is convenient to use the technology of homology of small categories. For any small category $I$ and ring $R$, we 
denote by $\operatorname{Fun}(I, R)$ the abelian category of functors from $I$ to $R$-modules, with the derived category $\mathcal{D}(I, R)$, and for any functor $\gamma: I \rightarrow I^{\prime}$ between small categories, we denote by $\gamma^{*}: \operatorname{Fun}\left(I^{\prime}, R\right) \rightarrow \operatorname{Fun}(I, R)$ the pullback functor. The functor $\gamma^{*}$ is exact, and it has a left and a right adjoint that we denote by $\gamma_{!}, \gamma_{*}: \operatorname{Fun}(I, R) \rightarrow \operatorname{Fun}\left(I^{\prime}, R\right)$. For any $E \in \operatorname{Fun}(I, R)$, the homology $H_{.}(I, E)$ of the category $I$ with coefficients in $E$ is by definition given by

$$
H_{i}(I, E)=L^{i} \tau_{!} E,
$$

where $\tau: I \rightarrow$ pt is the tautological projection to the point category pt.

The specific small category that we need is A. Connes' cyclic category $\Lambda$ of [C]. We do not reproduce here the full definition (see e.g. [L]), but we do recall that objects of $\Lambda$ correspond to cellular decompositions of the circle $S^{1}$, and morphisms correspond to homotopy classes of cellular maps of a certain type. We call 0 -cells vertices, and we call 1-cells edges. For any $n \geq 1$, there is exactly one decomposition with $n$ vertices and $n$ edges. The corresponding object in $\Lambda$ is denoted $[n]$, and we denote by $V([n])$ the set of its vertices. Any map $f:\left[n^{\prime}\right] \rightarrow[n]$ in $\Lambda$ induces a map $f: V\left(\left[n^{\prime}\right]\right) \rightarrow$ $V([n])$. For any $v \in V([n])$, the preimage $f^{-1}(v) \subset V\left(\left[n^{\prime}\right]\right)$ carries a natural total order. We have Aut $([n])=\mathbb{Z} / n \mathbb{Z}$, the cyclic group, so that for any $E \in \operatorname{Fun}(\Lambda, R), E([n])$ is naturally an $R[\mathbb{Z} / n \mathbb{Z}]$-module. Moreover, we have a natural embedding $j: \Delta^{o} \rightarrow \Lambda$, where as usual, $\Delta$ is the category of finite non-empty totally ordered sets, and $\Delta^{o}$ is the opposite category. To keep notation consistent with the embedding $j$, we denote by $[n] \in \Delta$ the set with $n$ elements.

By definition, the category $\operatorname{Fun}\left(\Delta^{o}, R\right)$ is category of simplicial $R$-modules, and for any $E \in \operatorname{Fun}\left(\Delta^{o}, R\right)$, we have the standard chain complex $C H .(E)$ with terms $C H_{i}(E)=E([i+1]), i \geq 0$, and the differential $d_{i}: C H_{i}(E) \rightarrow C H_{i-1}(E)$ given by

$$
d_{i}=\sum_{0 \leq j \leq i} \delta_{j}^{i}
$$

where $\delta^{\bullet}$ are the face maps. Moreover, we also have another complex $C H_{.}^{\prime}(E)$ with the same terms as $C H_{.}(E)$, but with the differential given by (1.4) with the summation extended over $j$ from 0 to $i-1$ (that is, we drop the last term). Then the complex $C H .(E)$ computes the homology $H_{.}\left(\Delta^{o}, E\right)$, while the complex $C H_{.}^{\prime}(E)$ is acyclic - in fact, canonically contractible.

Now, for any object $E \in \operatorname{Fun}(\Lambda, R)$, we have the simplicial object $j^{*} E \in$ $\operatorname{Fun}\left(\Delta^{o}, R\right)$, and it is well-known that the complexes (1.3) for $E([n]), n \geq 1$ 
fit together into a single bicomplex

$$
C H_{.}^{\prime}(E) \stackrel{\text { id }-\sigma^{\dagger}}{\longrightarrow} C H \cdot(E),
$$

where for any $[n] \in \Lambda, \sigma \in \operatorname{Aut}([n])$ is the generator of the cyclic group $\mathbb{Z} / n \mathbb{Z}$, and $\sigma^{\dagger}=(-1)^{n+1} \sigma$. We denote by $C H .(E)$ the total complex of the bicomplex (1.5). Furthermore, it is also well-known that the maps $B$ of Example 1.1 fit together into a single map $B: C H_{.}(E) \rightarrow C H .(E)[-1]$ that turns $C H .(E)$ into a mixed complex.

It will be convenient to recast this construction in a slightly different way. For any $[n] \in \Lambda$, denote by $\mathbb{K}$. $([n])$ the standard celluar chain complex computing the homology of the circle $S^{1}$ with respect to the decomposition correcponding to $[n]$. Then it turns out that $K$. is functorial with respect to morphisms in $\Lambda$, so that we obtain an exact sequence

$$
0 \longrightarrow \mathbb{Z} \stackrel{\kappa_{1}}{\longrightarrow} \mathbb{K}_{1} \longrightarrow \mathbb{K}_{0} \longrightarrow \mathbb{Z} \stackrel{\kappa_{0}}{\longrightarrow} 0
$$

in the category $\operatorname{Fun}(\Lambda, \mathbb{Z})$. For any $E \in \operatorname{Fun}(\Lambda, R)$, denote $\mathbb{K} .(E)=\mathbb{K}$. $\otimes E$. Then $\mathbb{K}$. $(E)$ is a mixed complex in $\operatorname{Fun}(\Lambda, R)$, with the map $B$ given by

$$
B=\left(\kappa_{1} \circ \kappa_{0}\right) \otimes \mathrm{id} .
$$

Now for any $E \in \operatorname{Fun}(\Lambda, R)$, denote by $c c$. $(E)$ the cokernel of the natural map (1.5). Then one can show that we have natural identifications

$$
C H_{\bullet}\left(j^{*} E\right) \cong c c .\left(\mathbb{K}_{0}(E)\right), \quad C H_{\bullet}^{\prime}\left(j^{*} E\right) \cong c c .\left(\mathbb{K}_{1}(E)\right),
$$

and the mixed complex $C H$. $(E)$ is then given by

$$
C H .(E) \cong c c .\left(\mathbb{K}_{.}(E)\right) \text {, }
$$

with the map $B$ induced by the map $B$ of (1.7).

Finally, observe that if we are given a complex $E$. in the category $\operatorname{Fun}(\Lambda, R)$, then we can apply $c c_{\mathbf{.}}(-)$ and $C H_{\mathbf{.}}(-)$ to $E_{\text {. termwise. We }}$ denote by $c c_{\bullet}\left(E_{\bullet}\right), C H$. $(E$. $)$ the sum-total complexes of the resulting bicomplexes. Explicitly, we have

$$
c c_{j}\left(E_{\bullet}\right)=\bigoplus_{n \geq 1} E_{j+n}([n])_{\sigma^{\dagger}}, \quad j \in \mathbb{Z}
$$

with the differential induced by the differential (1.4) and the differential in the complex $E$. 
1.3 Cyclic homology. We can now define periodic and co-periodic cyclic homology.

Definition 1.2. Assume given a ring $R$ and a complex $E$. in the category $\operatorname{Fun}(\Lambda, R)$. Then the cyclic homology complex $C C_{\bullet}\left(E_{\bullet}\right)$, the periodic cyclic homology complex $C P .\left(E_{\bullet}\right)$, the co-periodic cyclic homology complex $\overline{C P} .\left(E_{\bullet}\right)$, and the polynomial periodic cyclic homology complex $\mathrm{cp} \mathbf{\bullet}_{\bullet}\left(E_{\bullet}\right)$ are given by

$$
\begin{aligned}
C C_{\bullet}\left(E_{\bullet}\right) & =\operatorname{Exp}\left(C H_{\bullet}\left(E_{\bullet}\right)\right), \quad \\
\overline{C P}_{\bullet}\left(E_{\bullet}\right) & =\overline{\operatorname{Per}}\left(C H_{\bullet}\left(E_{\bullet}\right)\right), \quad\left(E_{\bullet}\right)=\operatorname{Per}\left(C H_{\bullet}\left(E_{\bullet}\right)\right), \\
c p_{\bullet}\left(E_{\bullet}\right) & =\operatorname{per}\left(C H_{\bullet}\left(E_{\bullet}\right)\right) .
\end{aligned}
$$

The periodic resp. co-periodic cyclic homology $H P_{\text {. }}\left(E_{\bullet}\right)$ resp. $\overline{H P} .\left(E_{\bullet}\right)$ is the homology of the complexes $C P .\left(E_{\bullet}\right)$ resp. $\overline{C P}$. $\left(E_{\bullet}\right)$.

We note that the first line in (1.10) is completely standard; it is the second line that defines new theories introduced in Ka5. The homology of the complex $C C_{.}\left(E_{\bullet}\right)$ is the usual cyclic homology $H C_{\bullet}\left(E_{\bullet}\right)$, and it is well-known that we have a natural identification

$$
H_{\text {. }}\left(E_{\bullet}\right) \cong H_{\bullet}\left(\Lambda, E_{\bullet}\right) \text {, }
$$

where $E$. in the right-hand side is understood as the corresponding object in the derived category $\mathcal{D}(\Lambda, R)$. One can combine this with (1.1) to express $H P .(-)$. Co-periodic cyclic homology functor $\overline{H P}$. (-) does not admit such a homological expression, and in fact, it is not true that quasiisomorphic complexes have isomorphic $\overline{H P}$. For any complex $E$., we do have functorial maps

$$
C P_{\bullet}\left(E_{\bullet}\right) \longleftarrow c p .\left(E_{\bullet}\right) \longrightarrow \overline{C P} .\left(E_{\bullet}\right)
$$

induced by the maps (1.2), but in general, these maps are not quasiisomorphisms. We also note that we have a natural functorial map

$$
\alpha: C C_{\bullet}\left(E_{\bullet}\right) \rightarrow c c .\left(E_{\bullet}\right),
$$

and in general, this map is not a quasiisomorphism either. One example where it is a quasiisomorphism is $E_{\text {. }}=\mathbb{K}$. $\left(E^{\prime}\right.$.) for some complex $E_{\text {. }}^{\prime}$ in $\operatorname{Fun}(\Lambda, R)$. In this case, by [Ka5, Lemma 3.11], $\alpha$ induces a natural isomorphism

$$
H C .\left(\mathbb{K}_{\bullet}\left(E_{\bullet}^{\prime}\right)\right) \cong H H_{\bullet}\left(E_{\bullet}^{\prime}\right),
$$


where $H_{\bullet} H_{\text {. }}\left(E_{\text {. }}^{\prime}\right)$ is the homology of the complex $C H_{\bullet}\left(E_{\text {. }}^{\prime}\right)$ (or equivalently, of the complex $\left.C H .\left(j^{*} E^{\prime}\right)\right)$.

Assume now given a Noetherian commutative ring $k$ and a DG algebra $A$. termwise-flat over $k$. Then one defines a complex $A^{\natural}$. in $\operatorname{Fun}(\Lambda, k)$ as follows. For any $[n] \in \Lambda, A^{\natural}([n]) \cong A^{\otimes_{k} n}$, with terms of the product numbered by vertices $v \in V([n])$. For any map $f:\left[n^{\prime}\right] \rightarrow[n]$, the corresponding map $A^{\natural}(f)$ is given by

$$
A_{\bullet}^{\natural}(f)=\bigotimes_{v \in V([n])} m_{f^{-1}(v)}
$$

where for any finite set $S, m_{S}: A_{\cdot}^{\otimes_{k} S} \rightarrow A$. is the multiplication map in the DG algebra $A$. . Then by definition, cyclic homology and periodic cyclic homology of the DG algebra $A$. are the cyclic homology and the periodic cyclic homology of the complex $A_{\text {. }}^{\natural}$, and we define co-periodic and polynomial periodic cyclic homology $\overline{H P}$. $\left(A_{\bullet}\right), h p .\left(A_{\bullet}\right)$ in the same way: we set

$$
\overline{H P} \cdot\left(A_{\bullet}\right)=\overline{H P} \cdot\left(A_{\bullet}^{\natural}\right), \quad h p_{\bullet}\left(A_{\bullet}\right)=h p .\left(A_{\bullet}^{\natural}\right) .
$$

Here are, then, the main two results about $\overline{H P}$. $\left(A_{\bullet}\right)$ proved in [Ka5].

Theorem 1.3. (i) Co-periodic cyclic homology functor $\overline{H P}$.(-) extends to an additive invariant of small DG categories (in particular, a quasiisomorphism fo DG algebras induces an isomorphism of their co-periodic cyclic homology groups).

(ii) Assume that $k \otimes \mathbb{Q}=0$ and $A$. is homologically smooth and homologically bounded over $k$. Then the maps (1.12) for $E_{.}=A_{\text {. }}^{\natural}$ are quasiisomorphisms, so that we have

$$
H P_{.}\left(A_{\bullet}\right) \cong h p .\left(A_{\bullet}\right) \cong \overline{H P} .\left(A_{\bullet}\right)
$$

Proof. The first statement (i) is [Ka5, Theorem 6.6], and the second statement (ii) is contained in [Ka5, Theorem 6.7].

In Theorem 1.3 (ii), homologically smooth as usual means that $A$. is

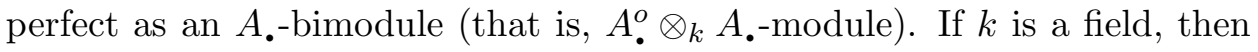
homologically bounded simply means that the complex $A$. has a finite number of non-trivial homology groups. In the general case, this must hold for $A . \otimes \bar{k}$, where $\bar{k}$ is any residue field of the ring $k$. 


\section{Conjugate spectral sequence.}

For any mixed complex $\left\langle V_{\bullet}, B\right\rangle$ in an abelian category $\mathcal{E}$, the $u$-adic filtration on $\operatorname{Per}\left(V_{\bullet}\right) \cong V_{\bullet}((u))$ induces a convergent spectral sequence

$$
H_{\bullet}\left(V_{\bullet}\right)((u)) \Rightarrow H_{\bullet}\left(\operatorname{Per}\left(V_{\bullet}\right)\right) \text {, }
$$

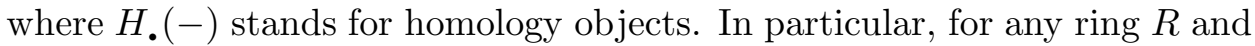
complex $E . \in \operatorname{Fun}(\Lambda, R)$, we have a convergent spectral sequence

$$
H H_{\bullet}\left(E_{\bullet}\right)((u)) \Rightarrow H P .\left(E_{\bullet}\right) \text {. }
$$

If $E_{\bullet}=A_{\text {. }}^{\natural}$ for a DG algebra $A_{\text {. }}$ over a commutative ring $k, H_{\bullet}\left(A_{\text {. }}^{\natural}\right)$ is naturally identified with the Hochschild homology $H H_{\text {. }}\left(A_{\text {. }}\right)$ of the DG algebra $A_{\text {. }}$, so that (2.1) reads as

$$
H_{\bullet}\left(A_{\bullet}\right)((u)) \Rightarrow H P .\left(A_{\bullet}\right) \text {. }
$$

This is the Hodge-to-de Rham spectral sequence.

For co-periodic cyclic homology, no analog of (2.1) is currently known, but under some assumptions, we do have a version of (2.2). This was inroduced in Ka5] under the name of the conjugate spectral sequence. In this section, we briefly recall the construction.

2.1 Filtrations. The main technical tool used in [Ka5] for studying coperiodic cyclic homology is the use of filtrations and filtered derived categories. Filtrations are decreasing and indexed by all integers - that is, a filtered complex in an abelian category $\mathcal{E}$ is a complex $E$. equipped with a collection of subcomplexes $F^{i} E . \subset E$., $i \in \mathbb{Z}$ such that $F^{i} E . \subset F^{j} E$. for $i \geq j$. A filtration $F^{\bullet}$ is termwise-split if for any $i$ and $j$, the embedding $F^{i} E_{j} \rightarrow E_{j}$ admits a one-sided inverse $E_{j} \rightarrow F^{i} E_{j}$. The stupid filtration $F^{\bullet}$ on a complex $E$. is obtained by setting $F^{j} E_{i}=E_{i}$ if $i+j \leq 0$ and 0 otherwise; it is tautologically termwise-split. For any filtration $F^{\bullet}$ and any integer $n \geq 1$, the $n$-th rescaling $F_{[n]}^{\cdot}$ of $F^{\bullet}$ is given by $F_{[n]}^{i}=F^{i n}, i \in \mathbb{Z}$, and the shift by $n$ $F_{n}^{\bullet}$ is given by $F_{n}^{i}=F^{i+n}$. A map $\left\langle E_{.}^{\prime}, F_{.}^{\prime}\right\rangle \rightarrow\left\langle E_{.}, F_{\bullet}\right\rangle$ of filtered complexes is a filtered quasiisomorphism if for any $i$, the induced map $\operatorname{gr}_{F^{\prime}}^{i} E^{\prime} \rightarrow \operatorname{gr}_{F}^{i} E$. of associated graded quotients is a quasiisomorphism (there is no require-

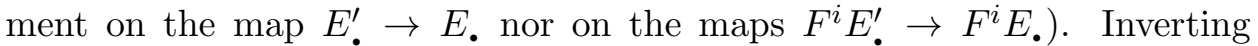
filtered quasiisomorphisms in the category of filtered complexes, we obtain the filtered derived category $\mathcal{D} F(\mathcal{E})$. 
The completion $\widehat{E}$. of a filtered complex $\left\langle E_{.}, F^{\bullet}\right\rangle$ is given by

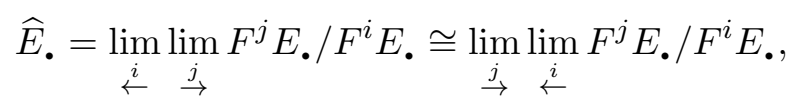

where the limit is over all integers $i \geq j$, with $i$ going to $\infty$ and $j$ to $-\infty$. If a map $E^{\prime} \rightarrow E$. is a filtered quasiisomorphism, the induced map $\widehat{E}^{\prime} \rightarrow \widehat{E}$. of completions is a quasiisomorphism. The converse is not true: two very different filtrations can have the same completion. Specifically, say that two filtrations $F_{1}^{\cdot}, F_{2}^{*}$ on the same complex $E$. are commensurable if for any integers $i, j$, there exist integers $j_{1} \leq j \leq j_{2}$ such that $F_{2}^{j_{2}} E_{i} \subset F_{1}^{j} E_{i} \subset$ $F_{2}^{j_{1}} E_{i}$ and $F_{1}^{j_{2}} E_{i} \subset F_{2}^{j} E_{i} \subset F_{1}^{j_{1}} E_{i}$. Then two commensurable filtrations on a complex $E$. obviously give the same completion $\widehat{E}$.

Every shift $F_{n}^{\cdot}$ and every rescaling $F_{[n]}^{\cdot}$ of a filtration $F^{\bullet}$ is obviously commensurable to $F^{\cdot}$. For a more non-trivial example, assume given a filtered complex $\left\langle E_{\bullet}, F^{\bullet}\right\rangle$, and define its filtered truncations by setting

$$
\begin{aligned}
& \tau_{\geq n}^{F} E_{i}=d^{-1}\left(F^{n+1-i} E_{i-1}\right) \cap F^{n-i} E_{i} \subset E_{i}, \\
& \tau_{\leq n}^{F} E_{i}=E_{i} /\left(F^{n+1-i} E_{i}+d\left(F^{n-i} E_{i+1}\right)\right)
\end{aligned}
$$

for any integer $n$. Denote also $\tau_{[n, m]}^{F}=\tau_{\geq n}^{F} \tau_{\leq m}^{F}$ for any integers $n \leq m$. Then the subcomplexes $\tau_{\geq n}^{F} E$. $\subset E$. for varying $n$ give a filtration $\tau^{\bullet}$ on $E_{\text {. }}$, and this filtration is commensurable to $F^{\bullet}$.

Example 2.1. Assume that $E$. is the sum-total complex of a bicomplex $E_{., .}$, and let $F^{\bullet}$ be the stupid filtration with respect to the first coordinate. Then $\tau_{\geq n}^{F} E_{\bullet}, \tau_{\leq n}^{F} E$. are canonical truncations with respect to the second coordinate.

In the general case, the truncation functors $\tau^{F}$ are also related to the canonical filtrations: for any integers $i, j$, we have natural isomorphisms

$$
\operatorname{gr}_{F}^{j} \tau_{\geq i}^{F} E_{\bullet} \cong \tau_{\geq i-j} \operatorname{gr}_{F}^{j} E_{\bullet}, \quad \operatorname{gr}_{F}^{j} \tau_{\leq i}^{F} E_{\bullet} \cong \tau_{\leq i-j} \operatorname{gr}_{F}^{j} E_{\bullet}
$$

where $\tau_{\geq n}, \tau_{\leq n}$ are the usual canonical truncations. In particular, the functors $\tau^{F}$ preserve filtered quasiisomorphisms and descend to the filtered derived category $\mathcal{D} F(\mathcal{E})$, where they become truncation functors with respect to the well-known $t$-structure of [BBD] (the heart of this $t$-structure is the category of complexes in $\mathcal{E}$ ). However, it will be useful to have the truncation functors already on the level of filtered complexes.

We note that since the filtrations $F^{\bullet}$ and $\tau^{\bullet}$ are commensurable, $\operatorname{gr}_{\tau}^{i} E$. is complete with respect to $F^{\bullet}$ for any integer $i$, so that for any filtered 
quaiisomorphism $f:\left\langle E_{.}^{\prime}, F_{.}^{\prime}\right\rangle \rightarrow\left\langle E_{.}, F_{.}\right\rangle$is also a filtered quasiisomorphism with respect to the filtrations $\tau$. Thus sending $\left\langle E_{\bullet}, F^{\bullet}\right\rangle$ to $\left\langle E_{\bullet}, \tau^{\bullet}\right\rangle$ descends to an endofunctor

$$
\mathcal{D} F(\mathcal{E}) \rightarrow \mathcal{D} F(\mathcal{E})
$$

of the filtered derived category $\mathcal{D} F(\mathcal{E})$. We also note that for any integer $i$ and filtered complex $\left\langle E_{\bullet}, F^{\bullet}\right\rangle, \operatorname{gr}_{\tau}^{i} E$. is quasiisomorphic (but not isomorphic) to $\tau_{[i, i]}^{F} E_{\text {. }}$.

Now, for any ring $R$ and any complex $E$. in the category $\operatorname{Fun}(\Lambda, R)$ equipped with a termwise-split filtration $F^{*}$, define the standard filtration on the complex $c c$. $(E$.) by setting

$$
F^{i} c c_{j}\left(E_{\bullet}\right)=\bigoplus_{n \geq 1} F^{i+n} E_{j+n}([n])_{\sigma^{\dagger}}, \quad i, j \in \mathbb{Z}
$$

where we use the decomposition (1.9) of the complex $c c .(E$.$) . By virtue of$ (1.8), the standard filtration extends to the complex $C H_{\bullet}\left(E_{\bullet}\right)$ and then to its periodic expansions $\overline{C P}$. $\left(E_{\bullet}\right), c p$. $\left(E_{\text {. }}\right)$.

Lemma 2.2 ([Ka5, Lemma 3.8]). Equip a complex $E$. in $\operatorname{Fun}(\Lambda, R)$ with the stupid filtration $F^{*}$. Then the co-periodic complex $\overline{C P} .\left(E_{0}\right)$ is isomorphic to the completion of the polynomial periodic complex cp. $\left(E_{\bullet}\right)$ with respect to the standard filtration.

By virtue of this result, one can reduce the study of $\overline{C P} .(E$. $)$ to the study of $c p .(E$.$) equipped with the standard filtration.$

Let us now do the following. Fix an integer $p \geq 1$, and for any complex $E$. in $\operatorname{Fun}(\Lambda, R)$ equipped with the stupid filtration, denote by $c p .(E)^{[p]}$ the complex $c p .(E$. $)$ equipped with the $p$-th rescaling of the standard filtration of (2.5).

Definition 2.3. The conjugate filtration $V^{\bullet}$ on $c p_{\bullet}\left(E_{\bullet}\right)$ is given by

$$
V^{n} c p .\left(E_{\bullet}\right)=\tau_{\geq 2 n-1} c p .\left(E_{\bullet}\right)^{[p]}, \quad n \in \mathbb{Z} .
$$

Note that since the complex $c p .(E$. $)$ is by definition 2-periodic, the conjugate filtration is periodic: we have

$$
V^{n} c p_{\bullet}\left(E_{\bullet}\right) \cong V^{0} c p .\left(E_{\bullet}\right)[2 n]
$$


for any integer $n$. By definition, the conjugate filtration is a shift of a rescaling of the filtration $\tau^{*}$, so that it is commensurable to the $p$-th rescaling of the standard filtration on $c p_{\text {. }}\left(E_{\text {. }}\right)$. This is in turn commensurable to the standard filtration itself. Therefore by Lemma 2.2, the co-periodic cyclic homology complex $\overline{C P}$. $\left(E_{\text {. }}\right)$ is isomorphic to the completion of the complex $c p .(E$. $)$ with respect to the conjugate filtration (2.6). We then have a convergent spectral sequence

$$
H_{\bullet}\left(\operatorname{gr}_{V}^{0} c p .\left(E_{\bullet}\right)\right)\left(\left(u^{-1}\right)\right) \Rightarrow \overline{H P} .\left(E_{\bullet}\right),
$$

where as before, $H_{\text {. }}(-)$ stands for homology objects, $u$ is a formal generator of cohomological degree 2, and we have used the identifications (2.7).

2.2 Edgewise subdivision. In general, the spectral sequence (2.8) does not seem to be particularly useful, since its initial term is rather obscure. However, under some assumptions, it can be computed explicitly. The first step in this computation is the so-called edgewise subdivision.

Recall that for any integer $l$, the category $\Lambda$ has a cousin $\Lambda_{l}$ corresponding to the $l$-fold cover $S^{1} \rightarrow S^{1}$. Objects in $\Lambda_{l}$ are objects $[n l] \in \Lambda, n \geq 1$, equipped with the order- $l$ automorphism $\tau=\sigma^{n}:[n l] \rightarrow[n l]$, and morphisms are morphisms in $\Lambda$ that commute with the automorphism $\tau$. It is convenient to number objects in $\Lambda_{l}$ by positive integers, so that $[n] \in \Lambda_{l}$ corresponds to $[n l] \in \Lambda$. We have the forgetful functor $i_{l}: \Lambda_{l} \rightarrow \Lambda,[n] \mapsto[n l]$, and we also have a natural projection $\pi_{l}: \Lambda_{l} \rightarrow \Lambda$ that sends $[n l]$ to the object $[n]$ considered as an induced cellular decomposition of $S^{1} \cong S^{1} / \tau$. The functor $\pi_{l}: \Lambda_{l} \rightarrow \Lambda$ is a Grothendieck bifibration with fiber $\mathrm{pt}_{l}$, the groupoid with one object with automorphism group $\mathbb{Z} / l \mathbb{Z}$. The functor $i_{l}$ induces the pullback functor $i_{l}^{*}: \operatorname{Fun}(\Lambda, R) \rightarrow \operatorname{Fun}\left(\Lambda_{l}, R\right)$, classically known as "edgewise subdivision functor", and the functor $\pi_{l}$ induces functors $\pi_{l !}, \pi_{l *}: \operatorname{Fun}\left(\Lambda_{l}, R\right) \rightarrow \operatorname{Fun}(\Lambda, R)$. We will need a slightly more complicated functor $\pi_{l b}$ that sends complexes in $\operatorname{Fun}\left(\Lambda_{l}, R\right)$ to complexes $\operatorname{Fun}(\Lambda, R)$. It is given by

$$
\pi_{l b} E_{\bullet}=\operatorname{per}\left(\pi_{l !}\left(i_{l}^{*} \mathbb{K}_{\bullet} \otimes E_{\bullet}\right)\right)
$$

Equivalently, one can use $\pi_{l *}$ - we have a natural trace map $\pi_{l !} \rightarrow \pi_{l *}$, and when evaluated on the complex $i_{l}^{*} \mathbb{K}$. $\otimes E_{\text {. }}$, this map is an isomorphism. The functors $i_{l}^{*}$ and $\pi_{l b}$ extend to filtered complexes in the obvious way.

Now assume that our base ring $R$ is annihilated by a prime $p$, and restrict our attention to the $p$-fold cover $\Lambda_{p}$. Then we have the following result. 
Proposition 2.4 ([Ka5, Proposition 4.4]). Assume given a complex E. in $\operatorname{Fun}(\Lambda, R)$ equipped with some filtration $F^{\cdot}$, and denote by $E_{\cdot}^{[p]}$ the same complex equipped with the $p$-the rescaling $F_{[p]}^{\cdot}$ of the filtration. Then there exists a functorial map

$$
\nu_{p}: c c \cdot\left(\pi_{p b} i_{p}^{*} E_{\bullet}^{[p]}\right) \rightarrow c p .\left(E_{\bullet}\right)^{[p]},
$$

and this map is a filtered quasiisomorphism with respect to the standard filtrations.

For the next step, we need to impose a condition on the complex $E$. Recall that we have assumed $p R=0$. Therefore the cohomology algebra $H^{\bullet}(\mathbb{Z} / p \mathbb{Z}, R)$ of the cyclic group $\mathbb{Z} / p \mathbb{Z}$ is given by

$$
H^{\bullet}(\mathbb{Z} / p \mathbb{Z}, R) \cong R[u]\langle\varepsilon\rangle,
$$

where $u$ is a generator of degree 2 , and $\varepsilon$ is a generator of degree 1 . The relations are: $u$ commutes with $\varepsilon$, and

$$
\varepsilon^{2}= \begin{cases}u, & p=2, \\ 0, & p \text { is odd }\end{cases}
$$

In particular, for any $R[\mathbb{Z} / p \mathbb{Z}]$-module $E$ and any integer $i$, we have a natural map

$$
\varepsilon_{i}: \check{H}_{i}(\mathbb{Z} / p \mathbb{Z}, E) \rightarrow \check{H}_{i-1}(\mathbb{Z} / p \mathbb{Z}, E),
$$

where $\check{H}$. $(\mathbb{Z} / p \mathbb{Z},-)$ is the Tate homology of $\mathbb{Z} / p \mathbb{Z}$. Since the Tate homology of the cyclic group is 2-periodic, we have $\varepsilon_{i}=\varepsilon_{i+2 n}$ for any $n$, so that $\varepsilon_{i}$ only depends on the parity of the integer $i$.

Definition 2.5. An $R[\mathbb{Z} / p \mathbb{Z}]$-module $E$ is tight if $\varepsilon_{1}$ is an isomorphism. A complex $E$. of $R[\mathbb{Z} / p \mathbb{Z}]$-modules is tight if $E_{i}$ is a tight $R[\mathbb{Z} / p \mathbb{Z}]$-module when $i$ is divisible by $p$ and a projective $R[\mathbb{Z} / p \mathbb{Z}]$-module otherwise. A complex $E$. in the category $\operatorname{Fun}\left(\Lambda_{p}, R\right)$ is tight if for any $[n] \in \Lambda_{p}, E$. $([n])$ is a tight complex with respect to the action of the group $\mathbb{Z} / p \mathbb{Z}$ generated by $\tau \in \operatorname{Aut}([n])$.

Note that if $p$ is odd, then (2.11) shows that for a tight $R[\mathbb{Z} / p \mathbb{Z}]$-module $E$, we have $\varepsilon_{0}=0$. Conversely, if $p=2, \varepsilon_{0}$ is also an isomorphism, and in fact the tightness condition is always satisfied (both for an object and for a complex). 
Lemma 2.6. (i) Assume given a tight complex $E$. of $R[\mathbb{Z} / p \mathbb{Z}]$-modules, and denote by $E^{[p]}$ the complex $E$. equipped with the p-th rescaling of the stupid filtration. Denote by $\check{C} .\left(\mathbb{Z} / p \mathbb{Z}, E_{\cdot}^{[p]}\right)$ the sum-total complex of the Tate homology bicomplex of the group $\mathbb{Z} / p \mathbb{Z}$ with coefficients in $E$. equipped with the filtration $F^{\bullet}$ induced by the filtration on $E_{\cdot}^{[p]}$. Let

$$
\mathrm{I}\left(E_{\bullet}\right)=\tau_{[0,0]}^{F} \check{C}_{\bullet}\left(\mathbb{Z} / p \mathbb{Z}, E_{\bullet}^{[p]}\right) .
$$

Then the induced filtration $F^{\cdot}$ on $\mathrm{I}\left(E_{\text {. }}\right)$ is the stupid filtration, and for any integer $i$, we have a natural filtered isomorphism

$$
\tau_{[i, i]}^{F} \check{C} .\left(\mathbb{Z} / p \mathbb{Z}, E_{\bullet}^{[p]}\right) \cong \mathrm{I}\left(E_{\bullet}\right)[i] .
$$

(ii) Assume given a tight complex $E$. in $\operatorname{Fun}\left(\Lambda_{p}, R\right)$, and denote by $E_{\cdot}^{[p]}$ the complex $E$. equipped with the p-th rescaling of the stupid filtration. Consider the induced filtration on the complex $\pi_{p b} E_{\cdot}^{[p]}$, and let $\mathrm{I}\left(E_{\bullet}\right)=$ $\tau_{[0,0]}^{F} \pi_{p b} E_{\bullet}^{[p]}$. Then for any $[n] \in \Lambda$, we have $\mathrm{I}\left(E_{\bullet}\right)([n]) \cong \mathrm{I}\left(E_{\bullet}([n])\right)$, and for any integer $[i]$, the isomorphisms (2.13) induce an isomorphism

$$
\tau_{[i, i]}^{F} \pi_{p b} E_{\bullet}^{[p]} \cong \mathrm{I}\left(E_{\bullet}\right)[i] .
$$

Proof. Almost all of the statements are obvious; the non-obvious ones are [Ka5, Lemma 5.3].

Explicitly, the isomorphisms (2.13) can be described as follows. By periodicity, we have an isomorphism

$$
u: \check{C} \cdot\left(\mathbb{Z} / p \mathbb{Z}, E_{\bullet}^{[p]}\right) \cong \check{C} \cdot\left(\mathbb{Z} / p \mathbb{Z}, E_{\bullet}^{[p]}\right)[2]
$$

corresponding to the action of the generator $u$ of the cohomology algebra (2.10). Twists by powers of $u$ provide isomorphisms (2.13) for even $i$. To obtain the isomorphisms for odd $i$, one considers the action of the generator $\varepsilon$. This gives natural maps

$$
\varepsilon_{i}: \tau_{[i, i]}^{F} \check{C} .\left(\mathbb{Z} / p \mathbb{Z}, E_{\bullet}^{[p]}\right) \rightarrow \tau_{[i-1, i-1]}^{F} \check{C} .\left(\mathbb{Z} / p \mathbb{Z}, E_{\bullet}^{[p]}\right),
$$

a filtered refinement of (2.12). Since $E$. is tight, $\varepsilon_{i}$ is a filtered isomorphism for any odd $i$.

All of this works relatively over the category $\Lambda$; in particular, we have natural maps

$$
\varepsilon_{i}: \tau_{[i, i]}^{F} \pi_{p b} E_{\bullet}^{[p]} \rightarrow \tau_{[i-1, i-1]}^{F} \pi_{p b} E_{\bullet}^{[p]}
$$

for any tight complex $E$. in $\operatorname{Fun}\left(\Lambda_{p}, R\right)$, and these maps are invertible for odd $i$. 
2.3 Localized conjugate filtration. Now, as it turns out, for a complex $E$. in $\operatorname{Fun}(\Lambda, R)$ with tight edgewise subdivision $i_{p}^{*} E$., we can localize the conjugate filtration (2.6) with respect to the category $\Lambda$ and express it in terms of the complex $\pi_{p b} i_{p}^{*} E$. Namely, introduce the following.

Definition 2.7. For any complex $E$. in $\operatorname{Fun}\left(\Lambda_{p}, R\right)$, the conjugate filtration $V^{\bullet}$ on the complex $\pi_{p b} E$. is given by

$$
V^{n} \pi_{p b} E_{\bullet}=\tau_{\geq 2 n}^{F} \pi_{p b} E_{\bullet}^{[p]},
$$

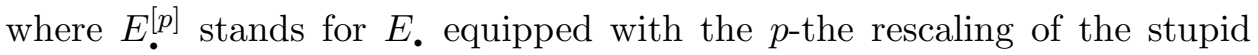
filtrarion.

We can then take a complex $E$. in $\operatorname{Fun}(\Lambda, R)$, consider the corresponding complex $\pi_{p b} i_{p}^{*} E$. equipped with the conjugate filtration (2.17), and apply to it the cyclic homology complex functor $C C$. (-). Since the functor $C C .(-)$ is exact, the conjugate filtration induces a filtration $V^{\bullet}$ on $C C_{\bullet}\left(\pi_{p b} i_{p}^{*} E_{\bullet}\right)$. We denote by $\widehat{C C} .\left(\pi_{p b} i_{p}^{*} E_{\bullet}\right)$ the completion of the complex $C C_{\bullet}\left(\pi_{p b} i_{p}^{*} E_{\bullet}\right)$ with respect to the filtration $V^{\bullet}$.

Consider now the composition

$$
C C_{\bullet}\left(\pi_{p b} i_{p}^{*} E_{\bullet}\right) \stackrel{\alpha}{\longrightarrow} c c_{\bullet}\left(\pi_{p b} i_{p}^{*} E_{\bullet}\right) \stackrel{\nu_{p}}{\longrightarrow} c p_{\bullet}\left(E_{\bullet}\right)
$$

of the map $\alpha$ of (1.13) and the natural map $\nu_{p}$ of Proposition 2.4.

Lemma 2.8. (i) Assume that the complex $i_{p}^{*} E$. in $\operatorname{Fun}\left(\Lambda_{p}, R\right)$ is tight in the sense of Definition 2.5. Then the map (2.18) extends to a quasiisomorphism

$$
\widehat{C C} .\left(\pi_{p b} i_{p}^{*} E_{\bullet}\right) \cong \overline{C P} .\left(E_{\bullet}\right) .
$$

(ii) Moreover, assume that the prime $p$ is odd. Then (2.18) itself is a filtered quasiisomorphism with respect to the filtrations $V^{\bullet}$ of (2.17) resp. (2.6).

(iii) In addition, still assuming that $p$ is odd, let $\mathrm{I}\left(i_{p}^{*} E_{\text {. }}\right)$ be canonical complex of Lemma 2.6. Then we have a natural isomorphism

$$
\operatorname{gr}_{V}^{0} \pi_{p b} i_{p}^{*} E . \cong \mathbb{K}\left(\mathrm{I}\left(i_{p}^{*} E_{\bullet}\right)\right)
$$

in the derived category $\mathcal{D}(\Lambda, R)$. 
Proof. (i) is [Ka5, Lemma 5.19], (ii) is [Ka5, Lemma 5.9], and (iii) is Ka5, Lemma 5.7].

By virtue of this result, for an odd prime $p$, we can rewrite the spectral sequence (2.8) as

$$
C C .\left(\operatorname{gr}_{V}^{0} \pi_{p b} i_{p}^{*} E_{\bullet}\right)\left(\left(u^{-1}\right)\right) \Rightarrow \overline{H P} .\left(E_{\bullet}\right),
$$

where we have used the obvious counterpart of the periodicity isomorphism (2.7) for the filtration (2.17). Moreover, by (1.11), (2.19), and (1.14), we can further rewrite (2.21) as

$$
H_{\bullet}\left(\mathrm{I}\left(i_{p}^{*} E_{\bullet}\right)\right)\left(\left(u^{-1}\right)\right) \Rightarrow \overline{H P} .\left(E_{\bullet}\right) \text {, }
$$

where as in (2.8), $u$ is a formal generator of cohomological degree 2.

2.4 DG algebras. To make the spectral sequence (2.21) useful, it remains to compute the complex $\mathrm{I}\left(i_{p}^{*} E\right.$. $)$. In order to do this, we need to assume further that $E$. comes from a DG algebra $A$. We thus assume given a commutative ring $k$ annihilated by an odd prime $p$. We denote by $k^{(1)}$ the Frobenius twist of $k$ - that is, $k$ considered as a module over itself via the absolute Frobenius map $k \rightarrow k$. For any flat $k$-module $V$, we denote $V^{(1)}=V \otimes_{k} k^{(1)}$.

Proposition 2.9 ([Ka5, Proposition 6.10]). Assume that the ring $k$ is Noetherian. Then for any complex $V$. of flat $k$-modules, the complex $V_{{ }^{\otimes}}^{\otimes_{k}} p$ of $k[\mathbb{Z} / p \mathbb{Z}]$-modules is tight in the sense of Definition [2.5, and we have a natural identification

$$
\mathrm{I}\left(V_{\cdot}^{\otimes_{k} p}\right) \cong V_{\cdot}^{(1)},
$$

where $\mathbf{I}(-)$ is the canonical complex provided by Lemma 2.6.

Corollary 2.10. Assume given a DG algebra A. termwise-flat over a commutative ring $k$ annihilated by an odd prime $p$. Then the complex $i_{p}^{*} A_{\text {. }}^{\natural}$ in the category $\operatorname{Fun}\left(\Lambda_{p}, k\right)$ is tight in the sense of Definition 2.5, and we have a natural identification

$$
\mathbf{I}\left(i_{p}^{*} A_{\bullet}^{\natural}\right) \cong\left(A_{\bullet}^{\natural}\right)^{(1)} .
$$

Proof. This is [Ka5, Lemma 6.19].

By virtue of this corollary, we have a natural identification

$$
H H_{\bullet}\left(\mathrm{I}\left(i_{p}^{*} A_{\bullet}^{\natural}\right)\right) \cong H H_{\bullet}^{(1)}\left(A_{\bullet}\right),
$$




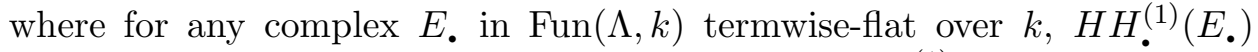
denotes the homology of the Frobenius twist $C H_{\mathbf{*}}\left(E_{\mathbf{*}}\right)^{(1)}$ of the Hochschild homology complex $C H$. $(E$.). If the commutative ring $k$ is finitely generated and regular, so that $k^{(1)}$ is flat over $k$, then we have

$$
H_{\bullet}^{(1)}\left(A_{\bullet}\right) \cong H_{\bullet}\left(A_{\bullet}^{(1)}\right),
$$

where $A_{0}^{(1)}$ is the Frobenius twist of the DG algebra $A_{\text {. }}$ Then (2.21) takes its final form - what we have is a spectral sequence

$$
H_{\bullet}\left(A_{\bullet}^{(1)}\right)\left(\left(u^{-1}\right)\right) \Rightarrow \overline{H P} \text {. }\left(A_{\bullet}\right) \text {. }
$$

This is the conjugate spectral sequence for the DG algebra $A_{\text {. }}$.

We finish the section with an alternative description of the complex $i_{p}^{*} A^{\natural}$. in terms of the $p$-tensor power algebra $A_{\bullet}^{\otimes_{k} p}$; this goes back to [Ka2, Subsection 2.2], and we will need it later in Section 3,

For any small category $\mathcal{C}$, sending an object $[n] \in \Lambda$ to $\mathcal{C}^{V([n])}$ defines a contravariant functor from $\Lambda$ to the category of small categories, and the Grothendieck construction [G] associates a category fibered over $\Lambda$ to this functor. We will denote the category by $\mathcal{C} 2 \Lambda$, and we will denote the fibration by $\pi: \mathcal{C} \prec \Lambda \rightarrow \Lambda$. Then for any DG algebra $A$. in the category $\operatorname{Fun}(\mathcal{C}, k)$ - or equivalently, for any functor from $\mathcal{C}$ to DG algebras over $k$ - the construction of the complex $A^{\natural}$. of Subsection 1.3 admits a straightforward refinement that produces a complex $A^{\natural}$. in the category $\operatorname{Fun}(\mathcal{C} \prec \Lambda, k)$.

Consider the case $\mathcal{C}=\mathrm{pt}_{p}$, the groupoid with one object with automorphism group $G=\mathbb{Z} / p \mathbb{Z}$. Then a DG algebra in $\operatorname{Fun}\left(\mathrm{pt}_{p}, k\right)$ is simply a DG algebra $A$. over $k$ equipped with an action of the group $G$, and what we obtain is a complex $A^{\natural}$. in the category $\operatorname{Fun}\left(\mathrm{pt}_{p} \imath \Lambda, k\right)$. The categories $\Lambda$ and $\mathrm{pt}_{p} \prec \Lambda$ have the same objects, and for any such object $[n]$, we have

$$
A_{\bullet}^{\natural}([n])=A_{\bullet}^{\otimes_{k} n},
$$

with the natural action of $G^{n}$. By base change [Ka2, Lemma 1.7], we have

$$
\pi_{*} A_{\bullet}^{\natural}([n]) \cong\left(A_{\bullet}^{\natural}([n])\right)^{G^{n}}=\bar{A}_{\bullet}^{\natural}([n]),
$$

where we denote by $\bar{A} .=A_{\text {. }}^{G} \subset A$. the subalgebra of $G$-invariants. Taken together, these identifications produce an isomorphism

$$
\pi_{*} A^{\natural} \cong \bar{A}_{.}^{\natural} .
$$


Now note that we also have a natural embedding

$$
\lambda: \Lambda_{p} \rightarrow \mathrm{pt}_{p} \prec \Lambda
$$

that induces the diagonal embedding $\mathrm{pt}_{p} \rightarrow \mathrm{pt}_{p}^{n}$ on the fiber over any object $[n] \in \Lambda$. Then for any DG algebra $A$. over $k$, we have natural isomorphism

$$
i_{p}^{*} A_{\bullet}^{\natural} \cong \lambda^{*}\left(A_{\bullet}^{\otimes_{k} p}\right)^{\natural},
$$

where in the right-hand side, the $p$-th power $A_{\bullet}^{\otimes_{k} p}$ is equipped with the natural $G$-action by the longest permutation.

\section{Tate cohomology.}

To analyze the conjugate spectral sequence (2.22), we need a more invariant definition of the functor $\pi_{p b}$ of (2.9). The relevant conceptual formalism is that of Tate cohomology.

3.1 Relative Tate cohomology. Assume given a ring $k$, a finite group $G$, and a bounded complex $E$. of $k[G]$-modules. Recall that the Tate cohomology of the group $G$ with coefficients in $E$. is given by

$$
\check{H}^{\bullet}\left(G, E_{\bullet}\right)=\operatorname{Ext}_{\mathcal{D}(k[G]) / \mathcal{D}^{p f}(k[G])}^{\bullet}(k, E \text { • }),
$$

where $k$ is the trivial $k[G]$-module, and $\mathcal{D}(k[G]) / \mathcal{D}^{p f}(k[G])$ is the quotient of the derived category $\mathcal{D}(k[G])$ of all $k[G]$-modules by its full subcategory $\mathcal{D}^{p f}(k[G]) \subset \mathcal{D}(k[G])$ spanned by perfect complexes of $k[G]$-modules. In order to compute Tate cohomology, it is convenient to introduce the following (we use the same notation and terminology as in [Ka6, Subsection 6.3]).

Definition 3.1. (i) Resolution data for a finite group $G$ is a pair $\nu=$ $\left\langle P ., I^{\bullet}\right\rangle$ of a left free resolution $P$. and a right free resolution $I^{\bullet}$ of the trivial $\mathbb{Z}[G]$-module $\mathbb{Z}$.

(ii) For any associative unital ring $k$, any bounded complex $E$. of $k[G]$ modules, and any resolution data $\nu$, the Tate cohomology complex of $G$ with coefficients in $E$. is given by

$$
\check{C}^{\bullet}\left(G, \nu, E_{\bullet}\right)=\left(E . \otimes \bar{P}_{\bullet} \otimes I^{\bullet}\right)^{G},
$$

where $\bar{P}$. is the cone of the augmentation map $P_{\bullet} \rightarrow \mathbb{Z}$. 
(iii) For any associative unital ring $k$, any bounded complex $E$. of $k[G]$ modules, and any resolution data $\nu$, the reduced Tate cohomology complex of $G$ with coefficients in $E$. is given by

$$
\check{C}_{r e d}^{\bullet}\left(G, \nu, E_{\bullet}\right)=\left(E_{\bullet} \otimes \widetilde{P}_{\bullet}\right)^{G}
$$

where $\widetilde{P}$. is the cone of the natural map $P . \rightarrow \mathbb{Z} \rightarrow I^{\bullet}$.

Remark 3.2. Here as well as elsewhere, we use homological and cohomological indexes for complexes interchangeably, with the convention that $E_{i}=E^{-i}$.

Resolution data form a category in the obvious way, and this category is connected (for example, every $\nu$ is obviously connected by a chain of maps to a functorial resolution data set $\nu^{\prime}$ obtained by taking bar resolutions). For any resolution data $\nu$, we have a natural map $\widetilde{P} . \rightarrow \bar{P} . \otimes I^{\bullet}$, and the induced map

$$
\check{C}_{\text {red }}^{\bullet}\left(G, \nu, E_{\bullet}\right) \rightarrow \check{C} \cdot\left(G, \nu, E_{\bullet}\right)
$$

of Tate cohomology complexes is a quasiisomorphism for any bounded complex $E_{\text {. }}$. Moreover, a map of resolution data induces a map of Tate complexes, and these maps are also quasiisomorphisms. Therefore we can drop $\nu$ from notation and obtain a well-defined object $\check{C}_{\bullet}\left(G, E_{\bullet}\right) \cong \check{C}_{\text {red }}\left(G, E_{\text {. }}\right)$ in the derived category of $k$-modules. Its cohomology modules are then canonically identified with Tate cohomology groups $\check{H}^{\bullet}(G, E$. $)$.

It is obvious from (3.1) that $\check{H}^{\bullet}(G, k)$ is an algebra, and for any bounded complex $E_{\text {. }} \check{H}^{\bullet}\left(G, E_{\text {. }}\right)$ is a module over $\check{H}^{\bullet}(G, k)$. To lift it to the dervied category level, one chooses resolution data $\nu=\left\langle P_{.}, I^{\bullet}\right\rangle$ that are multiplicative in the following sense: both $I^{\bullet}$ and $\bar{P}$. are DG algebras, and the natural maps $\mathbb{Z} \rightarrow I^{\bullet}, \mathbb{Z} \rightarrow \bar{P}$. are algebra maps. Multiplicative resolution data exist. For each multiplicative $\nu, \check{C}^{\bullet}(G, \nu, k)$ is a DG algebra, and $\check{C}^{\bullet}(G, \nu, E$. $)$ is a module over this DG algebra. Passing to the derived category, we obtain well-defined multiplication maps

$$
\check{C}^{\bullet}(G, k) \stackrel{\llcorner}{\otimes} \check{C}^{\bullet}\left(G, E_{\bullet}\right) \rightarrow \check{C}^{\bullet}\left(G, E_{\bullet}\right)
$$

that do not depend on the choice of the resolution data $\nu$.

Assume now given small categories $\mathcal{C}, \mathcal{C}^{\prime}$ and a functor $\pi: \mathcal{C}^{\prime} \rightarrow \mathcal{C}$. Assume further that $\pi$ is a Grothendieck bifibration in the sense of [G], and that the fibers of this bifibrations are equivalent to $\mathrm{pt}_{G}$, the groupoid with 
one object with automorphism group $G$. By base change [Ka2, Lemma 1.7], for any object $c^{\prime} \in \mathcal{C}^{\prime}$ with $c=\pi\left(c^{\prime}\right) \in \mathcal{C}$, and any functor $E \in \operatorname{Fun}\left(\mathcal{C}^{\prime}, k\right)$, we have natural isomorphisms

$$
\pi_{!} E\left(c^{\prime}\right) \cong E\left(c^{\prime}\right)_{G}, \quad \pi_{*} E(c) \cong E\left(c^{\prime}\right)^{G},
$$

where $G$ acts on $E\left(c^{\prime}\right)$ via the embedding pt $_{G} \rightarrow \mathcal{C}^{\prime}$ of the fiber over $c \in \mathcal{C}$. Then one can obviously make the constructions above work "relatively over $\mathcal{C}$ ". Namely, one defines resolution data for $\pi$ as pairs $\nu=\left\langle P ., I^{\bullet}\right\rangle$ of a left and a right resolution of the constant functor $\mathbb{Z} \in \operatorname{Fun}\left(\mathcal{C}^{\prime}, \mathbb{Z}\right)$ such that for any $c \in \mathcal{C}, \nu$ restricted to the fiber $\mathrm{pt}_{G} \subset \mathcal{C}^{\prime}$ over $c$ gives resolution data in the sense of Definition 3.1. One shows easily that resolution data exist (e.g. take the bar resolutions) and that the category of resolution data is connected. Then for any resolution data $\nu$, one defines

$$
\pi_{\nu}^{b}(E)=\pi_{*}\left(E \otimes \bar{P} . \otimes I^{\bullet}\right), \quad \pi_{\nu}^{b b}(E)=\pi_{*}\left(E \otimes \widetilde{P}_{\bullet}\right),
$$

where $\bar{P}$. is as in (3.2), and $\widetilde{P}$. is as in (3.3). By (3.4), we have a natural quasiisomorphism $\pi_{\nu}^{b}(E) \cong \pi_{\nu}^{b b}(E)$, and both complexes do not depend on the choice of $\nu$. All in all, we obtain a well-defined object

$$
\pi^{b}(E) \in \mathcal{D}(\mathcal{C}, k)
$$

in the derived category $\mathcal{D}(\mathcal{C}, k)$. For any object $c^{\prime} \in \mathcal{C}^{\prime}$ with $c=\pi\left(c^{\prime}\right) \in \mathcal{C}$, (3.4) gives a natural identification

$$
\pi^{b}(E)(c) \cong \check{C}^{\bullet}\left(G, E\left(c^{\prime}\right)\right) .
$$

Moreover, as in Ka6, Subsection 6.3], one can choose multiplicative resolution data for $\pi$. This shows that $\pi^{b}(k)$ is an algebra object in $\mathcal{D}(\mathcal{C}, k)$, and for any $E$, we have natural action maps

$$
\pi^{b}(k) \stackrel{\llcorner}{\otimes} \pi^{b}(E) \rightarrow \pi^{b}(E)
$$

that turn $\pi^{b}(E)$ into a module object over the algebra $\pi^{b}(k)$.

Finally, we observe that all of the above can be repeated verbatim for a bounded complex $E$. instead of a single object $E$ in $\operatorname{Fun}\left(\mathcal{C}^{\prime}, k\right)$. Moreover, assume given a filtered complex $\left\langle E_{\bullet}, F^{\bullet}\right\rangle$ in $\operatorname{Fun}\left(\mathcal{C}^{\prime}, k\right)$, and assume that the filtration $F^{\bullet}$ is termwise-split, and $\operatorname{gr}_{F}^{i} E$. is bounded for any integer $i$. Then again, (3.5) gives an object

$$
\pi^{b} E . \in \mathcal{D} F(\mathcal{C}, k)
$$

in the filtered derived category $\mathcal{D} F(\mathcal{C}, k)$, and as such, it does not depend on the choice of the resolution data $\nu$. 
3.2 Conjugate filtration. We now want to apply relative Tate cohomology to the study of the conjugate filtration of Definition 3.2. We assume that the base ring $k$ is commutative and annihilated by a prime $p$. For simplicity, we also assume right away that $k$ is finitely generated and regular, so that the absolute Frobenius map $k \rightarrow k$ is finite and flat. Now, consider the natural projection

$$
\pi_{p}: \Lambda_{p} \rightarrow \Lambda \text {. }
$$

This is a Grothendieck bifibration, and its fiber is $\mathrm{pt}_{p}$, the groupoid with one object with automorphism group $\mathbb{Z} / p \mathbb{Z}$. Therefore for any filtered complex $\left\langle E_{\bullet}, F^{\bullet}\right\rangle$ in $\operatorname{Fun}\left(\Lambda_{p}, k\right)$ such that the filtration $F^{\bullet}$ is termwise-split and $\operatorname{gr}_{F}^{i} E$. is bounded for any $i$, (3.7) provides a natural object

$$
\pi_{p}^{b} E . \in \mathcal{D} F(\Lambda, k) .
$$

We note that we have a natural isomorphism

$$
\pi_{\nu}^{b b} E_{.} \cong \pi_{p b} E_{.}[1],
$$

where $\pi_{p b} E$. is the complex of (2.9), and $\nu$ is the pair of resolutions of $\mathbb{Z}$ obtained by periodization of the complex $\mathbb{K}$. of (1.6). Therefore the object (3.8) coincides with $\pi_{p b} E$. up to a homological shift.

In particular, let $E$. be an arbitrary complex in $\operatorname{Fun}\left(\Lambda_{p}, k\right)$, and let $E^{[p]}$ be $E$. equipped with the $p$-th rescaling of the stupid filtration. Then all the assumptions on the filtration are satisfied, so that we have a well-defined object $\pi_{p}^{b} E^{[p]}$ in $\mathcal{D} F(\Lambda, k)$. We also have the action map

$$
\pi_{p}^{b} k \stackrel{\llcorner}{\otimes} \pi_{p}^{b} E^{[p]} \rightarrow \pi_{p}^{b} E^{[p]}
$$

induced by the map (3.6) .

Lemma 3.3 ([Ka4, Lemma 3.2]). We have a canonical isomorphism

$$
\pi_{p b} k \cong \mathbb{K} .(k)((u))=\bigoplus_{i \in \mathbb{Z}} \mathbb{K} .(k)[2 i]
$$

of complexes in $\operatorname{Fun}(\Lambda, k)$.

By (3.9), this gives an isomorphism $\tau_{[i, i]} \pi_{p}^{b} k \cong k[i]$ for any integer $i$. Since the filtered truncation functors (2.3) are obviously compatible with the tensor products, the map (3.10) then induces a map

$$
\varepsilon_{i}: \tau_{[1,1]} \pi_{p}^{b} k \otimes \tau_{[i, i]}^{F} \pi_{p}^{b} E_{\bullet}^{[p]} \cong \tau_{[i, i]}^{F} \pi_{p}^{b} E_{\cdot}^{[p]}[1] \rightarrow \tau_{[i+1, i+1]}^{F} \pi_{p}^{b} E_{\bullet}^{[p]}
$$


for any integer $i$. These are exactly the maps (2.16). In particular, $\varepsilon_{i}$ only depends on the parity of $i$, and if $E$. is tight in the sense of Definition 2.5, then $\varepsilon_{1}$ is an isomorphism. In this case, for any integer $j$, (3.10) also induces an isomorphism

$$
\mathrm{I}\left(E_{\bullet}\right)[j]=\tau_{[j, j]} \pi_{b}^{p} k \otimes \tau_{[0,0]}^{F} \pi_{p}^{b} E_{\bullet}^{[p]} \cong \tau_{[j, j]}^{F} \pi_{p}^{b} E_{\bullet}^{[p]},
$$

a version of the isomorphism (2.13).

If $p$ is odd, tightness of $E$. further implies that $\varepsilon_{0}=0$. One immediate corollary of this is a short construction of the quasiisomorphism (2.19) of Lemma 2.8, for odd $p$. Indeed, since $\varepsilon_{2}=\varepsilon_{0}=0$ and the filtered truncations are multiplicative, the map (3.10) induces a natural map

$$
\begin{aligned}
\mathbb{K}\left(\mathrm{I}\left(E_{\bullet}\right)\right)[1] & =\mathbb{K}_{\bullet}[1] \otimes \mathrm{I}\left(E_{\bullet}\right) \cong \tau_{[1,2]} \pi_{p}^{b} k \otimes \tau_{[0,0]}^{F} \pi_{p}^{b} E_{\bullet}^{[p]} \rightarrow \\
& \rightarrow \tau_{[1,2]}^{F} \pi_{p}^{b} E_{b}^{[p]} \cong \operatorname{gr}_{V}^{0} \pi_{p b} E_{\bullet}[1],
\end{aligned}
$$

and since the maps (3.12) are isomorphisms, this map is a quasiisomorphism.

However, we will need another corollary. Namely, keep the assumption that $E$. is tight, let

$$
\bar{\pi}_{p}^{b} E_{.}=\tau_{\geq 0}^{F} \pi_{p}^{b} E_{\bullet}^{[p]},
$$

and consider this complex as an object in the filtered derived category $\mathcal{D} F(\Lambda, k)$ by equipping it with the filtration $\tau^{\bullet}$, via the functor (2.4). We then have the augmentation map

$$
a: \bar{\pi}_{p}^{b} E_{\bullet} \rightarrow \tau_{[0,0]} \pi_{p}^{b} E_{\bullet}^{[p]}=\mathrm{I}\left(E_{\bullet}\right)
$$

in $\mathcal{D} F(\Lambda, k)$, where $\mathrm{I}\left(E_{\bullet}\right)$ is placed in filtered degree 0 .

Lemma 3.4. Assume that the map a of (3.14) admits a one-sided inverse $s: \mathrm{I}\left(E_{\bullet}\right) \rightarrow \bar{\pi}_{p}^{b} E_{\bullet}, s \circ a=\mathrm{id}$ in the filtered derived category $\mathcal{D} F(\Lambda, k)$. Then the spectral sequence (2.21) degenerates.

Proof. By definition, we have a natural embedding $\bar{\pi}_{p}^{b} E . \rightarrow \pi_{p}^{b} E$. Composing it with $s$, we obtain a map

$$
\mathrm{I}\left(E_{.}\right) \rightarrow \pi_{p}^{b} E_{\bullet}
$$

and by (3.10), this map induces a map

$$
b: \pi_{p}^{b} k \otimes \mathrm{I}\left(E_{\bullet}\right) \cong \pi_{p}^{b} E_{\bullet} .
$$

For any integer $i$, the associated graded quotient $\operatorname{gr}_{\tau}^{i}(b)$ is the isomorphism (2.14) of Lemma 2.6, so that $b$ is a filtered quasiisomorphism. By the direct sum decomposition (3.11), this implies that the conjugate filtration on $\pi_{b}^{p} E$. splits. 
3.3 Splitting for DG algebras. Now keep the assumptions of the previous subsection, and assume given a DG algebra $A$. termwise-flat over the commutative ring $k$. Recall that by Corollary 2.10 , the corresponding complex $i_{p}^{*} A^{\natural}$. is tight in the sense of Definition 2.5, so that Lemma 3.4 applies. To finish the section, we prove one corollary of this fact.

Choose resolution data $\nu=\left\langle P_{.}, I^{\bullet}\right\rangle$ for the group $G=\mathbb{Z} / p \mathbb{Z}$ that are multiplicative, so that for any DG algebra $B$. termwise-flat over $k$ and equipped with a $G$-action, the Tate cohomology complex $\check{C} .(G, B$.$) is a$ DG algebra over $k$. Consider the $p$-fold tensor product $A^{\otimes_{k} p}$, and let $\mathbb{Z} / p \mathbb{Z}$ act on it by the longest permutation. Moreover, equip this tensor product with the $p$-the rescaling of the stupid filtration.

Definition 3.5. The DG algebra $P$. $(A$. $)$ is given by

$$
P_{\bullet}\left(A_{\bullet}\right)=\tau_{\geq 0}^{F} \check{C}^{\bullet}\left(\mathbb{Z} / p \mathbb{Z}, \nu, A_{\bullet}^{\otimes_{k} p}\right),
$$

where $A_{\bullet}^{\otimes_{k} p}$ is equipped with the $p$-th rescaling of the stupid filtration.

As for the complex (3.13), we equip $P .(A$.$) with the filtration \tau^{\bullet}$ and treat it as a filtered DG algebra. Since the filtered truncation functors $\tau_{\geq}^{F}$. are multiplicative, $P_{.}\left(A_{\bullet}\right)$ is well-defined, and up to a filtered quasiisomorphism, it does not depend on the choice of resolution data $\nu$. By Proposition [2.9, we have

$$
\operatorname{gr}_{\tau}^{i} P_{.}\left(A_{\bullet}\right) \cong A_{\bullet}^{(1)}[i]
$$

for any integer $i \geq 0$. In particular, we have an augmentation map

$$
a: P_{\bullet}\left(A_{\bullet}\right) \rightarrow A_{\bullet}^{(1)},
$$

and it is a filtered DG algebra map (where $A_{\bullet}^{(1)}$ is in filtered degree 0).

Proposition 3.6. Assume that the prime $p$ is odd, and that there exists a filtered $D G$ algebra $A^{\prime}$. over $k$ and a filtered $D G$ algebra map $s: A_{\bullet}^{\prime} \rightarrow A_{.}^{(1)}$ such that the composition $s \circ a: A^{\prime} \rightarrow A_{\cdot}^{(1)}$ is a filtered quasiisomorphism. Then the spectral sequence (2.22) for the DG algebra $A$. degenerates.

Proof. Fix multiplicative resolution data $\left\langle P_{.}, I^{\bullet}\right\rangle$ for the group $G=\mathbb{Z} / p \mathbb{Z}$, and consider the cone $\bar{P}$. of the augmentation map $P$. $\rightarrow \mathbb{Z}$. By definition, both $I^{\bullet}$ and $\bar{P}$. are DG algebras over $k$ equipped with a $G$-action.

For every integer $n \geq 1$, the complex $\bar{P}^{\otimes n}$ is concentrated in non-negative homological degrees, its degree- 0 term is $\mathbb{Z}$, while all the other terms are free 
$\mathbb{Z}[G]$-modules. Therefore $\bar{P}^{\otimes n}=\bar{P}^{n}$. for some free left resolution $P^{n}$ of the trivial module $\mathbb{Z}$. The complex $I^{\bullet \otimes n}$ is a right free resolution of $\mathbb{Z}$, so that $\left\langle P^{n}, I^{\bullet} \otimes n\right\rangle$ also gives resolution data for $G$ in the sense of Definition 3.1 ,

Now, since both $\bar{P}$. and $I^{\bullet}$ are DG algebras equipped with a $G$-action, we have natural complexes $\bar{P}^{\natural},\left(I^{\bullet}\right)^{\natural}$ in the category $\operatorname{Fun}\left(\mathrm{pt}_{p} \prec \Lambda, k\right)$. Restricting them to $\Lambda_{p} \subset \mathrm{pt}_{p}<\Lambda$ with respect to the embedding (2.25), we obtain complexes

$$
\bar{P}^{\lambda}=\lambda^{*} \bar{P}^{\natural}, \quad I_{\lambda}^{\bullet}=\lambda^{*}\left(I^{\bullet}\right)^{\natural}
$$

in the category $\operatorname{Fun}\left(\Lambda_{p}, k\right)$, and for any object $[n] \in \Lambda_{p}$, we have natural identifications

$$
\bar{P}_{.}^{\lambda}([n]) \cong \bar{P}^{\otimes n}, \quad I_{\lambda}^{\bullet}([n]) \cong I^{\bullet \otimes n} .
$$

Thus we can put together resolution data $\left\langle P_{.}^{n}, I^{\bullet} \otimes n\right\rangle, n \geq 1$ for the group $G$ into resolution data $\left\langle P_{\cdot}^{\lambda}, I_{\lambda}^{\cdot}\right\rangle$ for the bifibration $\pi_{p}: \Lambda_{p} \rightarrow \Lambda$ such that $\overline{P^{\lambda}}$. $\cong \bar{P}^{\lambda}$, and these resolution data can be then used for computing the relative Tate cohomology functor $\pi_{p}^{b}$. This gives a natural identification

$$
\pi_{p}^{\mathrm{b}} i_{p}^{*} A^{\natural} \cong \pi_{p *}\left(i_{p}^{*} A^{\natural} \otimes \bar{P}^{\lambda} \cdot \otimes I_{\lambda}^{\cdot}\right) .
$$

Now denote $B_{\bullet}=A_{\bullet}^{\otimes_{k} p} \otimes \bar{P} . \otimes I^{\bullet}$, and consider it as a $G$-equivariant DG algebra over $k$. Then by virtue of (2.26), we can rewrite (3.16) as

$$
\pi_{p}^{b} i_{p}^{*} A^{\natural} \cong \pi_{p *} \lambda^{*} B^{\natural} .
$$

Note that we have a natural map

$$
\pi_{*} B^{\natural} \rightarrow \pi_{p *} \lambda^{*} B_{\bullet}^{\natural},
$$

where $\pi: \mathrm{pt}_{p} \imath \Lambda \rightarrow \Lambda$ is the natural fibration. At each object $[n] \in \Lambda$, we can evaluate $\pi_{*} B^{\natural}$. by (2.23), and then this map is just the natural embedding

$$
\left(B_{\bullet}^{G}\right)^{\otimes_{k} n}=\left(B_{\bullet}^{\otimes_{k} n}\right)^{G^{n}} \rightarrow\left(B_{\bullet}^{\otimes_{k} n}\right)^{G} .
$$

Moreover, by (2.24) and (3.17), the map (3.18) actually gives a natural map

$$
\bar{B}_{.}^{\natural} \cong \pi_{*} B^{\natural} \rightarrow \pi_{p}^{\mathrm{b}} i_{p}^{*} A_{\bullet}^{\natural}
$$

where $\bar{B} . \subset B$. is the subalgebra of $G$-invariants.

Now equip $A_{\bullet}^{\otimes_{k} p}$ with the $p$-the rescaling of the stupid filtration, and consider the corresponding filtrations on the algebras $B_{.}, \bar{B}$. Then since $\tau_{\geq 0}^{F}$ is a multiplicative functor, the natural map (3.19) induces a map

$$
\left(\tau_{\geq 0}^{F} \bar{B} .\right)^{\natural} \rightarrow \tau_{\geq 0}^{F} \pi_{p}^{b} i_{p}^{*} A_{.}^{\natural}=\bar{\pi}_{b}^{p} i_{p}^{*} A_{.}^{\natural}
$$


By construction, if we compose this map with the projection

$$
\bar{\pi}_{p}^{b} i_{p}^{*} A_{\bullet}^{\natural} \rightarrow \operatorname{gr}_{\tau}^{0} \bar{\pi}_{p}^{b} i_{p}^{*} A_{\bullet}^{\natural} \cong \mathrm{I}\left(i_{p}^{*} A_{\bullet}^{\natural}\right) \cong A_{\bullet}^{(1) \natural},
$$

then the resulting map is induced by the augmentation map

$$
\tau_{\geq 0}^{F} \bar{B} . \rightarrow \tau_{[0,0]}^{F} \bar{B} . \cong A_{\bullet}^{(1)} .
$$

It remains to spell out the notation. By definition, we actually have

$$
\bar{B} .=\check{C}_{\bullet}\left(G, \nu, A^{\otimes_{k} n}\right),
$$

where $\nu$ stands for our original resolution data $\left\langle P_{.}, I^{\bullet}\right\rangle$. Therefore $\tau_{\geq 0}^{F}(\bar{B}.) \cong$ $P$. (A.), and the map (3.20) is a map

$$
P .\left(A_{\bullet}\right)^{\natural} \rightarrow A_{\natural}^{(1)} .
$$

If there exists a filtered DG algebra $A^{\prime}$. and a filtered DG algebra map $s: A_{\text {. }}^{\prime} \rightarrow P_{\text {. }}\left(A_{\text {. }}\right)$ satisfying the assumptions of the Proposition, then we also have a filtered map $A_{\bullet}^{\prime} \rightarrow P_{\bullet}\left(A_{\bullet}\right)^{\natural}$, and the composition map

$$
A_{\bullet}^{\prime} \longrightarrow P_{\bullet}\left(A_{\bullet}\right) \longrightarrow \bar{\pi}_{p}^{\dagger} i_{p}^{*} A_{\bullet}^{\natural} \longrightarrow A_{\bullet}^{(1) \natural}
$$

is a filtered quasiisomorphism. Then we are done by Lemma 3.4 .

\section{Characteristic 2.}

We now make a digression and explain how to modify the arguments of [Ka5] to obtain the conjugate spectral sequence (2.22) in characteristic 2 . The problem here is Lemma 2.8, while (i) is true in any characteristic, (ii) is definitely wrong in characteristic 2, and it is currently unknown whether (iii) is true or not. However, there is the following weaker result.

Proposition 4.1. For any DG algebra A. over a perfect field $k$ of positive characteristic $p$, there exists a natural isomorphism

$$
H C \cdot\left(\operatorname{gr}_{V}^{0} \pi_{p b} i_{p}^{*} A_{\bullet}^{\natural}\right) \cong H H_{\bullet}\left(A_{\bullet}^{(1)}\right) .
$$

While weaker than (2.19), this identification still allows one to rewrite (2.20) in the form (2.22), at least for DG algebras over a perfect field. For degeneration questions, this is irrelevant; the reader who is only integerested in degeneration of the spectral sequences can safely skip this section. 
4.1 Trace functors. To get a better handle on the complex $\pi_{p b} i_{p}^{*} A_{\text {. }}^{\natural}$, we use the formalism of trace functors of [Ka3, Section 2]. Here are the basic ingredients.

One starts by "categorifying" the construction of the object $A^{\natural}$ of Subsection 1.3. To every small monoidal category $\mathcal{C}$, one associates a covariant functor from $\Lambda$ to the category of small categories that sends $[n] \in \Lambda$ to $\mathcal{C}^{V([n])}$, and sends a morphism $f:\left[n^{\prime}\right] \rightarrow[n]$ to the product of multiplication functors $m_{f^{-1}(v)}: \mathcal{C}^{f^{-1}(v)} \rightarrow \mathcal{C}, v \in V([n])$ induced by the monoidal structure on $\mathcal{C}$, as in (1.15). Applying the Grothendieck construction, one obtains a category $\mathcal{C}^{\natural}$ and a cofibration $\rho: \mathcal{C}^{\natural} \rightarrow \Lambda$. This is somewhat similar to the wreath product construction $\mathcal{C}<\Lambda$, except that the functor is covariant, not contravariant, and the projection $\rho: \mathcal{C}^{\natural} \rightarrow \Lambda$ is a cofibration, not a fibration.

Explicitly, objects of $\mathcal{C}^{\natural}$ are pairs $\left\langle[n],\left\{c_{v}\right\}\right\rangle$ of an object $[n] \in \Lambda$ and a collection $\left\{c_{v}\right\}$ of objects in $\mathcal{C}$ numbered by vertices $v \in V([n])$. A morphism from $\left\langle\left[n^{\prime}\right],\left\{c_{v}^{\prime}\right\}\right\rangle$ to $\left\langle[n],\left\{c_{v}\right\}\right\rangle$ is given by a morphism $f:\left[n^{\prime}\right] \rightarrow[n]$ and a collection of morphisms

$$
f_{v}: \bigotimes_{v^{\prime} \in f^{-1}(v)} c_{v^{\prime}}^{\prime} \rightarrow c_{v}, \quad v \in V([n])
$$

A morphism is cartesian if all the components $f_{v}$ are invertible. Note that stated in this way, the definition makes perfect sense even when the category $\mathcal{C}$ is not small.

Definition 4.2. A trace functor from a monoidal category $\mathcal{C}$ to some category $\mathcal{E}$ is a functor $F: \mathcal{C}^{\natural} \rightarrow \mathcal{E}$ that sends cartesian maps in $\mathcal{C}^{\natural}$ to invertible maps in $\mathcal{E}$.

Explicitly, a trace functor is given by a functor $F: \mathcal{C} \rightarrow \mathcal{E}$ and a collection of isomorphisms

$$
\tau_{M, N}: F(M \otimes N) \cong F(N \otimes M), \quad M, N \in \mathcal{C}
$$

satisfying some compatibility constraints (see [Ka3, Subsection 2.1]). A trivial example of a trace functor is obtained by fixing a commutative ring $k$, and taking $\mathcal{C}=\mathcal{E}=k$-mod, the category of of $k$-modules, with $\tau_{-,-}$being the standard commutativity isomorphisms. There are also non-trivial examples. One such was considered in $[\mathrm{Ka} 3$ in detail. We still take $\mathcal{C}=\mathcal{E}=k$-mod, fix an integer $l \geq 1$, and let

$$
F(V)=V_{\sigma}^{\otimes_{k} l}
$$


where $\sigma: V^{\otimes_{k} l} \rightarrow V^{\otimes_{k} l}$ is the order-l permutation. The maps (4.2) are given by $\tau_{M, N}=\tau_{M, N}^{\prime} \circ\left(\sigma_{M} \otimes \mathrm{id}\right)$, where $\tau_{M, N}^{\prime}$ are the commutativity maps, and $\sigma_{M}$ is the order- $n$ permutation acting on $M^{\otimes_{k} l}$.

Every algebra object $A$ in the monoidal category $\mathcal{C}$ defines a section $\alpha: \Lambda \rightarrow \mathcal{C}^{\natural}$ of the cofibration $\rho: \mathcal{C}^{\natural} \rightarrow \Lambda$, and composing this section with a trace functor $F$ gives a natural functor

$$
F A^{\natural}=F \circ \alpha: \Lambda \rightarrow \mathcal{E} .
$$

If $\mathcal{C}=\mathcal{E}=k$-mod, what we obtain is an object $F A^{\natural}$ in the category $\operatorname{Fun}(\Lambda, k)$ associated to any associative unital algebra $A$ over $k$. If $F$ is the identity functor with the tautological teace functor structure, then this just the object $A^{\natural}$ of Subsection 1.3. In general, we obtain a version of cyclic homology twisted by the trace functor $F$, the main object of study in [Ka3].

Another way to express this is to say that a trace functor $F: \mathcal{C} \rightarrow k$-mod defines an object $F^{\natural} \in \operatorname{Fun}\left(\mathcal{C}^{\natural}, k\right)$, and we have $F A^{\natural}=\alpha^{*} F^{\natural}$. Analogously, a trace functor from $\mathcal{C}$ to the category $C$. $(k)$ of complexes of $k$-modules gives a complex $F^{\natural}$ in $\operatorname{Fun}\left(\mathcal{C}^{\natural}, k\right)$, and $F A^{\natural}=\alpha^{*} F^{\natural}$ is a complex in $\operatorname{Fun}(\Lambda, k)$.

Remark 4.3. Strictly speaking, when $\mathcal{C}$ is not small, $\operatorname{Fun}\left(\mathcal{C}^{\natural}, k\right)$ is not a well-defined category (Hom-sets might be large). A convenient solution is to only consider functors that commute with filtered colimits. Then each such functor from say $k$-mod to $k$-mod is completely determined by its restriction to the full subcategory spanned by finitely generated projective $k$-modules, and since this category is small, the problem does not arise. The same works for complexes (and the subcategory of perfect complexes). In our example, all large monoidal categories will be of this sort, so we will adopt this point of view. By abuse of notation, we will still use notation of the form $\operatorname{Fun}\left(\mathcal{C}^{\natural}, k\right)$ for the category for functors that commute with filtered colimits.

Let us now construct the cyclic power trace functor (4.3) more canonically. Fix a monoidal category $\mathcal{C}$ and an integer $l \geq 1$, and define a category $\mathcal{C}_{l}^{\natural}$ by the cartesian square

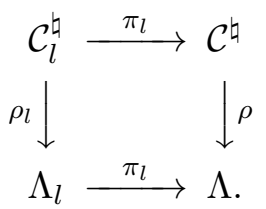


Then the functor $i_{l}: \Lambda_{l} \rightarrow \Lambda$ fits into a commutative square

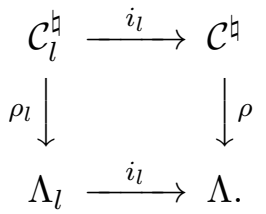

Explicitly, $\mathcal{C}_{l}^{\natural}$ is the category of pairs $\left\langle[n],\left\{c_{v}\right\}\right\rangle,[n] \in \Lambda_{l}, c_{v} \in \mathcal{C}, v \in V([n])$. Then the top arrow in (4.5) is the functor sending a sequence $\left\{c_{v}\right\}, v \in V([n])$ to the same sequence repeated $l$ times. In particular, it sends cartesian maps to cartesian maps.

We now observe that for any trace functor $F$ from $\mathcal{C}$ to the category of $k$ modules, with the corresponding object $F^{\natural} \in \operatorname{Fun}\left(\mathcal{C}^{\natural} \cdot k\right)$, the objects $\pi_{l !} i_{l}^{*} F^{\natural}$, $\pi_{l *} i_{l}^{*} F^{\natural}$ also correspond to trace functors from $\mathcal{C}$ to $k$-mod. If $\mathcal{C}=k$-mod and $F$ is the tautological functor, $\pi_{l !} i_{l}^{*} F^{\natural}$ corresponds to the cyclic power trace functor (4.3).

4.2 Quotients of the conjugate filtration. We now fix a perfect field $k$ of characteristic $p$, and we let $\mathcal{C}=C$. $(k)$ be the category of complexes of $k$-vector spaces. Moreover, denote by $I$. the complex in $\operatorname{Fun}\left(\mathcal{C}^{\natural}, k\right)$ corresponding to the identity trace functor $C .(k) \rightarrow C .(k)$, and denote by $I_{.}^{[p]}$ the complex $I$. equipped with the $p$-the rescaling of the stupid filtration.

Then the projection $\pi_{p}: \mathcal{C}_{p}^{\natural} \rightarrow \mathcal{C}^{\natural}$ of (4.4) is a bifibration with fiber $\mathrm{pt}_{p}$, so that we can consider relative Tate cohomology functor $\pi_{p}^{b}$. By base change [Ka2, Lemma 1.7], we have $\rho^{*} \circ \pi_{p}^{b} \cong \pi_{p}^{b} \circ \rho^{*}$, so that (3.11) yields a direct sum decomposition

$$
\pi_{p}^{b} k \cong \rho^{*} \mathbb{K} .(k)[1]((u))=\bigoplus_{i \in \mathbb{Z}} \rho^{*} \mathbb{K} .(k)[2 i+1]
$$

in the derived category $\mathcal{D}\left(\mathcal{C}^{\natural}, k\right)$. We also have the multiplication map (3.10) and all that it entails - in particular, the isomorphisms (3.12) for complexes $E$. in $\operatorname{Fun}\left(\mathcal{C}_{p}^{\natural}, k\right)$ that are tight in the obvious sense. We note that the pullback $i_{p}^{*} I$. of the tautological complex $I$. is tight.

Now fix some resolution data for $\pi_{p}$, so that $\pi_{p}^{b}$ is defined as a complex, and consider the complex

$$
C_{.}=\tau_{[0,1]}^{F} \pi_{p}^{b} i_{p}^{*} I_{\bullet}^{[p]}
$$

in the category $\operatorname{Fun}\left(\mathcal{C}^{\natural}, k\right)$. It must correspond to some trace functor from $\mathcal{C}=C$. $(k)$ to itself. Explicitly, the trace functor sends a complex $V$. to the 
complex

$$
C_{\bullet}\left(V_{\bullet}\right)=\tau_{[0,1]}^{F} \check{C}^{\bullet}\left(\mathbb{Z} / p \mathbb{Z}, V_{\bullet}^{\otimes_{k} p}\right),
$$

where we equip $V_{\bullet}^{\otimes_{k} p}$ with the $p$-th rescaling of the stupid filtration. By Proposition 2.9, we have a natural sequence

$$
0 \longrightarrow V_{\bullet}^{(1)}[1] \stackrel{b}{\longrightarrow} C_{\bullet}\left(V_{\bullet}\right) \stackrel{a}{\longrightarrow} V_{\bullet}^{(1)} \longrightarrow 0
$$

of functorial complexes of $k$-vector spaces that is quasiexact in the sense of Ka6, Definition 1.2] — this means that $a \circ b=0$, the map $a$ is surjective, the map $b$ is injective, and the complex $\operatorname{Ker} a / \operatorname{Im} b$ is acyclic. The map $a$ corresponds to a map

$$
a: C . \rightarrow I_{\bullet}^{(1)}
$$

in the category $\operatorname{Fun}\left(\mathcal{C}^{\natural}, k\right)$, where $I_{\bullet}^{(1)}$ is the Frobenius twist of the tautological complex $I_{\text {. }}$.

Moreover, consider the ring $W_{2}(k)$ of second Witt vectors of the field $k$, and let $\mathcal{C}_{1}=C .\left(W_{2}(k)\right)$ be the category of complexes of flat $W_{2}(k)$ modules. Denote by $q: \mathcal{C}_{1} \rightarrow \mathcal{C}$ the quotient functor sending a complex $V$. to its quotient $V . / p$. Note that $\mathcal{C}_{1}$ is a monoidal category, and $q$ is a monoidal functor. Moreover, extend (4.7) to $W_{2}(k)$-modules by setting

$$
C .\left(V_{\bullet}\right)=\tau_{[0,1]}^{F} \check{C}^{\bullet}\left(\mathbb{Z} / p \mathbb{Z}, V_{\bullet}^{\otimes W_{2}(k)}{ }^{p}\right)
$$

for any complex $V . \in \mathcal{C}_{1}$, where as in (4.7), we equip $V_{\cdot}^{\otimes_{W_{2}(k)}{ }^{p}}$ with the $p$-th rescaling of the stupid filtration. Then we have the following somewhat surprising result.

Lemma 4.4. (i) For any complex $V . \in \mathcal{C}_{1}$, we have a short exact sequence of complexes

$$
0 \longrightarrow C_{\bullet}\left(V_{\bullet} / p\right) \stackrel{p}{\longrightarrow} C_{\bullet}\left(V_{\bullet}\right) \stackrel{q}{\longrightarrow} C_{\bullet}\left(V_{\bullet} / p\right) \longrightarrow 0,
$$

where $p$ stands for multiplication by $p$, and $q$ is the quotient map.

(ii) For any $V_{.} \in \mathcal{C}$, let $\bar{C}$. ( $\left.V_{\bullet}\right)$ be the kernel of the map a of (4.8), and for any $V_{\bullet} \in \mathcal{C}_{1}$, let $\widetilde{C}_{\bullet}\left(V_{\bullet}\right)=C_{\bullet}\left(V_{\bullet}\right) / p \bar{C} .\left(V_{\bullet} / p\right)$. Then the composition map

$$
\widetilde{C} \cdot\left(V_{\bullet}\right) \stackrel{q}{\longrightarrow} C_{\bullet}\left(V_{\bullet} / p\right) \stackrel{a}{\longrightarrow} V_{\bullet}^{(1)}
$$

is a quasiisomorphism, and the functor $\widetilde{C_{.}}: \mathcal{C}_{1} \rightarrow \mathcal{C}_{1}$ factors through the quotient functor $q: \mathcal{C}_{1} \rightarrow \mathcal{C}$. 
Proof. (i) is [Ka6, Lemma 6.9], and (ii) is [Ka6, Proposition 6.11].

We note that $\widetilde{C} .(-)$ is the cokernel of a map of trace functors, thus itself inherits the structure of a trace functor. Then Lemma 4.4 (ii) implies that this trace functor is actually defined on $\mathcal{C}$ - namely, we have the following.

Corollary 4.5. Let $\widetilde{C}$. be the complex in $\operatorname{Fun}\left(\mathcal{C}_{1}^{\natural}, W_{2}(k)\right)$ corresponding to the trace functor $\widetilde{C} .(-)$ of Lemma 4.4 (ii). Then there exists a complex $W_{.} \in \operatorname{Fun}\left(\mathcal{C}, W_{2}(k)\right)$ such that

$$
\widetilde{C} . \cong q^{*} W_{.},
$$

where $q: \mathcal{C}_{1}^{\natural} \rightarrow \mathcal{C}^{\natural}$ is induced by the monoidal quotient functor $q: \mathcal{C}_{1} \rightarrow \mathcal{C}$.

Proof. The quotient functor $q$ is surjective on isomorphism classes of objects, so that it suffices to prove that the action of morphisms in $\mathcal{C}_{1}^{\natural}$ on $\widetilde{C}$. canonically factors through $q$. For cartesian morphisms, this is clear, so it suffices to check it for morphisms in the categories $\mathcal{C}_{1}^{V}([n]),[n] \in \Lambda$. This immediately follows from Lemma 4.4 (ii).

Proof of Proposition 4.1. Every $k$-vector space can be considered as a $W_{2}(k)$ module via the quotient map $W_{2}(k)$, so that we have a natural functor from $k$-vector sapces to $W_{2}(k)$-module. Moreover, for any small category $I$, we can apply this pointwise and obtain a functor

$$
\xi: \operatorname{Fun}(I, k) \rightarrow \operatorname{Fun}\left(I, W_{2}(k)\right) .
$$

This functor is exact and fully faitful. It induces a functor $\xi: \mathcal{D}(I, k) \rightarrow$ $\mathcal{D}\left(I, W_{2}(k)\right)$, and for any complex $E$. in $\operatorname{Fun}(I, k)$, we have a natural quasiisomorphism

$$
H_{\bullet}\left(I, \xi\left(I_{\bullet}\right)\right) \cong \xi\left(H_{\bullet}\left(I, E_{\bullet}\right)\right) .
$$

In particular, we can take $I=\Lambda$. Then by (1.11) and (1.14), to construct an isomorphism (4.1), it suffices to construct a functorial isomorphism

$$
\xi\left(\operatorname{gr}_{V}^{0} \pi_{p b} i_{p}^{*} A_{\bullet}^{\natural}\right) \cong \xi\left(\mathbb{K}\left(A_{\bullet}^{(1) \natural}\right)\right)
$$

in the derived category $\mathcal{D}\left(\Lambda, W_{2}(k)\right)$.

Consider first the universal situation. Denote

$$
R_{.}=\tau_{[1,2]}^{F} \pi_{p}^{b} i_{p}^{*} I_{\cdot}^{[p]}[-1],
$$


and recall that we have the multiplication map (3.10) for the bifibration $\pi_{p}: \mathcal{C}_{p}^{\natural} \rightarrow \mathcal{C}^{\natural}$. This map is compatible with filtered truncations, so by (4.6), it yields a map

$$
\rho^{*} \mathbb{K} .(k) \otimes_{k} C . \cong \tau_{[1,2]} \pi_{p}^{b} k[-1] \otimes_{k} \tau_{[0,1]}^{F} \pi_{p}^{b} i_{p}^{*} I_{\bullet}^{[p]} \rightarrow \tau_{[1,2]} \pi_{p}^{b} i_{p}^{*} I_{\bullet}^{[p]}[-1]=R_{\bullet} .
$$

This map induces a map

$$
\rho^{*} \mathbb{K}_{\bullet} \otimes \xi\left(C_{\bullet}\right) \rightarrow \xi\left(R_{\bullet}\right) .
$$

On the other hand, we have the complex $W$. of Corollary 4.5 and the natural

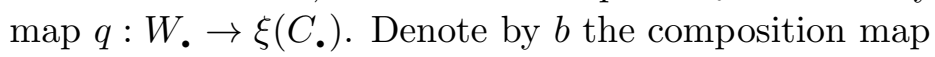

$$
\rho^{*} \mathbb{K} . \otimes W_{.} \stackrel{\text { id } \otimes q}{\longrightarrow} \rho^{*} \mathbb{K}_{\bullet} \otimes \xi\left(C_{\bullet}\right) \longrightarrow \xi\left(R_{\bullet}\right) .
$$

Then $b$ is filtered, hence compatible with the filtration $\tau^{\bullet}$. Moreover, since the composition $W_{\bullet} \rightarrow \xi\left(C_{\bullet}\right) \rightarrow \xi\left(I_{\bullet}^{(1)}\right)$ is a quasiisomorphism by Lemma 4.4, the source and the target of the map $b$ only have two non-trivial associated graded quotients $\operatorname{gr}_{\tau}^{i}$, for $i=0$ and 1, and in both cases, $\operatorname{gr}_{\tau}^{i}(b)$ is one of the isomorphisms (3.12) for the tight complex $I_{\cdot}^{[p]}$. Therefore $b$ is an isomorphism in the derived category $\mathcal{D}\left(\mathcal{C}^{\natural}, W_{2}(k)\right)$.

Now let $\alpha: \Lambda \rightarrow \mathcal{C}^{\natural}$ be the section of the projection $\rho: \mathcal{C}^{\natural} \rightarrow \Lambda$ corresponding to the DG algebra $A_{\text {. }}$, and consider the induced isomorphism

$$
\alpha^{*}(b): \mathbb{K}_{\bullet}\left(\alpha^{*} W_{\bullet}\right) \cong \alpha^{*}\left(\rho^{*} \mathbb{K}_{\bullet} \otimes W_{\bullet}\right) \rightarrow \alpha^{*} \xi\left(R_{\bullet}\right) .
$$

Then the right-hand side is exactly $\xi\left(\operatorname{gr}_{V}^{0} \pi_{p b} i_{p}^{*} A_{\text {. }}^{\natural}\right.$, and the left-hand side is naturally isomorphic to $\mathbb{K}_{.}\left(\alpha^{*} \xi\left(I_{\bullet}^{(1)}\right)\right) \cong \mathbb{K} .\left(\xi\left(A_{\bullet}^{(1) \natural}\right)\right) \cong \xi\left(\mathbb{K} .\left(A_{\bullet}^{(1) \natural}\right)\right)$.

\section{Degeneration.}

We now turn to degeneration results for the spectral sequences for cyclic homology. There are two statements: one for the conjugate spectral sequence (2.22), and one for the Hodge-to-de Rham spectral sequence (2.2).

5.1 Conjugate degeneration. Recall that for any field $k$, a square-zero extension $A^{\prime}$. of a DG algebra $A$. over $k$ by an $A$.-bimodule $M$. is a filtered DG algebra $\left\langle A_{\bullet}^{\prime}, \tau^{\bullet}\right\rangle$ such that $\tau^{0} A_{\bullet}^{\prime}=A_{.}^{\prime}, \tau^{2} A^{\prime}=0, \operatorname{gr}_{\tau}^{0} A_{\bullet}^{\prime} \cong A_{\bullet}$, and $\operatorname{gr}_{\tau}^{1} A^{\prime}$. is quasiisomorphic to $M_{\text {. }}$ as a bimodule over $\operatorname{gr}_{\tau}^{0} A_{\text {. }}^{\prime} \cong A_{\text {. }}$. Recall also that 
up to a quasiisomorphisms, square-zero extensions are classified by elements in the reduced Hochschild cohomology group

$$
\overline{H H}^{2}\left(A_{\bullet}, M_{\bullet}\right)=\operatorname{Ext}_{A_{\bullet} \otimes A_{\bullet}}\left(I_{\bullet}, M_{\bullet}\right),
$$

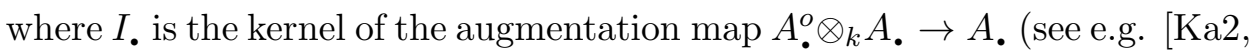
Subsection 4.3] but the claim is completely standard). Reduced Hochschild cohomology groups are related to the usual ones by the long exact sequence

$$
\overline{H H}^{\bullet}\left(A_{\bullet}, M_{\bullet}\right) \longrightarrow H H^{\bullet}\left(A_{\bullet}, M_{\bullet}\right) \longrightarrow M_{\bullet} \longrightarrow
$$

In particular, if $\overline{H H}^{2}\left(A_{\bullet}, M_{\bullet}\right)=0$ for some $M_{\text {. }}$, then every square-zero extension $A^{\prime}$. of $A$. by $M$. splits - there exists a DG algebra $A^{\prime \prime}$. and a map $A_{\text {. }}^{\prime \prime} \rightarrow A^{\prime}$. such that the composition map $A^{\prime \prime} \rightarrow A^{\prime} \rightarrow A$. is a quasiisomorphism.

Now fix a perfect field $k$ of some positive characteristic $p=$ char $k$, and assume given a DG algebra $A$. over $k$.

Theorem 5.1. Assume that the DG algebra A. over the field $k$ satisfies the following two properties:

(i) There exist a DG algebra $\widetilde{A}$. over the second Witt vectors ring $W_{2}(k)$ and a quasiisomorphism $\widetilde{A} . \stackrel{\llcorner}{\otimes}_{W_{2}(k)} k \cong A_{\text {. }}$.

(ii) The reduced Hochschild cohomology $\overline{H H}^{i}($. $)$ vanishes for $i \geq 2 p$.

Then the conjugate spectral sequence (2.22) degenerates at first trem, so that there exists an isomorphism $\overline{H P} .\left(A_{\text {. }} \cong H_{\text {. }}\left(A_{\bullet}^{(1)}\right)\left(\left(u^{-1}\right)\right)\right.$.

Remark 5.2. By Proposition 4.1, the spectral sequence (2.22) also exists for $p=2$, and Theorem 5.1 holds in this case, too. Of course in this case, the condition (ii) is pretty strong.

Proof. By Proposition [3.6, it suffices to construct a filtered DG algebra $A^{\prime}$. over $k$ and a filtered map $A_{\text {. }}^{\prime} \rightarrow P_{\text {. }}\left(A_{\text {. }}\right)$ such that the composition map $A_{\text {. }} \rightarrow A_{\text {. }}^{(1)}$ is a filtered quasiisomorphism (the assumption " $p$ is odd" in Proposition 3.6 is only needed to insure that the conjugate spectral sequence is well-defined, and it is not used in the proof).

To define the DG algebra $P .(A$.$) , we need to choose multiplicative res-$ olution data for the group $G=\mathbb{Z} / p \mathbb{Z}$, and we are free to do it in any way we like. Note that the $G$-action on the DG algebra $A_{\bullet}^{\otimes_{k} p}$ extends to the 
action of the symmetric group $\Sigma_{p}$. In particular, we have an action of the normalizer $\widehat{G}=(\mathbb{Z} / p \mathbb{Z}) \rtimes(\mathbb{Z} / p \mathbb{Z})^{*} \subset \Sigma_{p}$ of $G \subset \Sigma_{p}$. Choose some multiplicative resolution data for $\widehat{G}$, and restrict it to $G \subset G$. Then the resulting DG algebra $P .(A$.$) carries a natural action of (\mathbb{Z} / p \mathbb{Z})^{*}=\widehat{G} / G$. This action preserves the filtration $\tau^{*}$, and the augmentation map

$$
a: P_{.}\left(A_{\bullet}\right) \rightarrow \operatorname{gr}_{\tau}^{0} P_{.}\left(A_{\bullet}\right) \cong A_{\bullet}^{(1)}
$$

is $(\mathbb{Z} / p \mathbb{Z})^{*}$-invariant. For $i \geq 1$, we have the isomorphisms (3.15) induced by the isomorphisms (2.13); invariantly, they can be written as

$$
\operatorname{gr}_{\tau}^{i} P .\left(A_{\bullet}\right) \cong A_{\bullet}^{(1)}[i] \otimes_{k} \check{H}^{-i}(G, k),
$$

where the group $(\mathbb{Z} / p \mathbb{Z})^{*}$ acts on the right-hand side via its action on the Tate comology group $\check{H}^{-i}(G, k)$.

Now, the cohomology $H^{\bullet}(G, k)$ is given by (2.10). The group $(\mathbb{Z} / p \mathbb{Z})^{*}$ acts trivially on the generator $\varepsilon$, and it acts on the generator $u$ via its standard one-dimensional representation given by the action on $\mathbb{Z} / p \mathbb{Z} \subset k$. Therefore we have

$$
\check{H}^{-i}(G, k)^{(\mathbb{Z} / p \mathbb{Z})^{*}} \cong k
$$

if $i=0,1 \bmod 2(p-1)$, and 0 otherwise. We conclude that if we denote

$$
\bar{P} .\left(A_{\bullet}\right)=P \text {. }\left(A_{\bullet}\right)^{(\mathbb{Z} / p \mathbb{Z})^{*}} \subset P_{\bullet}\left(A_{\bullet}\right)
$$

and equip this DG algebra with the filtration induced by $\tau^{\bullet}$, then we have

$$
\operatorname{gr}_{\tau}^{i} \bar{P} .\left(A_{\bullet}\right) \cong \begin{cases}A_{\bullet}^{(1)}[i], & i=0,1 \bmod 2(p-1), \\ 0, & \text { otherwise. }\end{cases}
$$

If we denote by $e: \bar{P}$. $\left(A_{\text {. }}\right) \rightarrow P$. $\left(A_{\text {. }}\right)$ the embedding map, then $\operatorname{gr}_{\tau}^{i}(e)$ is a quasiisomorphism for $i=0,1 \bmod 2 p$, and $\operatorname{gr}_{\tau}^{i}(e)=0$ otherwise.

To prove degeneration, it suffices to construct a filtered DG algebra $A^{\prime}$. and a filtered map $s: A^{\prime} \rightarrow \bar{P}$. $\left(A_{\text {. }}\right)$, since then we can simply compose it with the embedding $e$. Moreover, a filtered algebra $\left\langle A_{\bullet}^{\prime}, \tau^{\bullet}\right\rangle$ is completely defined by its quotients $A_{\bullet}^{n}=A^{\prime} . / \tau^{n+1} A_{.}^{\prime}, n \geq 0$, together with the quotient maps $r_{n}: A^{n+1} \rightarrow A^{n}$, and by assumption, $A^{0}$. must be identified with the Frobenius twist $A_{\bullet}^{(1)}$ of the DG algebra $A_{\text {.. }}$ Thus if we denote

$$
P_{\bullet}^{n}=\bar{P}_{\bullet}\left(A_{\bullet}\right) / \tau^{n+1} \bar{P}_{\text {. }}\left(A_{\bullet}\right), \quad n \geq 1,
$$

and denote by $p_{n}: P_{.}^{n+1} \rightarrow P_{.}^{n}$ the quotient maps, then it suffices to construct a collection of DG algebras $A^{n}$. over $k$ for all $n \geq 1$, equipped with DG algebra maps $r_{n}: A_{\bullet}^{n+1} \rightarrow A_{\bullet}^{n}, s_{n}: A_{\bullet}^{n} \rightarrow P_{\bullet}^{n}$ such that 
- for every $n \geq 1$, we have $s_{n} \circ r_{n}=p_{n} \circ s_{n+1}$, and the composition map $a \circ e \circ s_{n}: A_{\bullet}^{n} \rightarrow A_{\bullet}^{(1)}$ is a quasiisomorphism.

We use induction on $n$. To start it, we take $n=1$; we need to find a DG algebra $A_{\text {. }}^{1}$ over $k$ and a map $s_{1}: A^{1} \rightarrow P^{1}$. such that $a \circ e \circ s_{1}: A^{1} \rightarrow A_{\text {. }}^{(1)}$ is a quasiisomorphism. By [Ka6, Proposition 6.13], this is possible precisely because the DG algebra $A$. satisfies the assumption (i) of the Theorem.

For the induction step, assume given $A_{\bullet}^{n-1}$ and $s_{n-1}: A_{\bullet^{n-1}} \rightarrow P_{\bullet^{n-1}}$, and consider the DG algebra $A_{\text {. }}^{\prime \prime}$ defined by the cartesian square

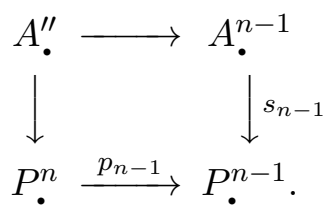

Then up to a quasiisomorphism, $A_{\text {. }}^{\prime \prime}$ is a square-zero extension of $A_{\bullet}^{(1)}$ by $\operatorname{gr}_{\tau}^{n} \bar{P}$. (A.), and finding $A_{\text {. }}^{n}$ with the maps $s_{n}, r_{n-1}$ satisfying $(\bullet)$ is equivalent to finding a DG algebra $A_{\text {. }}^{n}$ and a map $A_{\text {. }}^{n} \rightarrow A_{\text {. }}^{\prime \prime}$ such that the composition map

$$
A_{\bullet}^{n} \longrightarrow A_{\bullet}^{\prime \prime} \longrightarrow A_{\bullet}^{(1)}
$$

is a quasiisomorphism. In other words, we have to split the extension $A_{\text {. }}^{\prime \prime}$. The obstruction to doing this lies in the reduced Hochschild cohomology group $\overline{H H}^{2}\left(A_{\bullet}^{(1)}, \operatorname{gr}_{\tau}^{n} \bar{P} .\left(A_{\bullet}\right)\right)$. By (5.2) , this group vanishes unless $i=0,1$ $\bmod 2 p$, and in this case, we have

$$
\overline{H H}^{2}\left(A_{\bullet}^{(1)}, \operatorname{gr}_{\tau}^{n} \bar{P} \cdot\left(A_{\bullet}\right)\right) \cong \overline{H H}^{2+n}\left(A_{\bullet}^{(1)}\right) .
$$

Since $n \geq 2$ and $n=0,1 \bmod 2(p-1)$, we have $n \geq 2(p-1)$, and then this reduced Hochschild cohomology group vanishes by the assumption (ii).

Remark 5.3. The condition (ii) of Theorem 5.1 is slightly unnatural: while Hochschild homology and the conjugate spectral sequence are derived Morita-invariant, reduced Hochschild cohomology groups are not (because of the third term in (5.1)). One would like to have the same statement but with $H H^{\bullet}(-)$ instead of $\overline{H H}^{\bullet}(-)$. The simplest way to obtain such a statement would be to repeat the whole argument for DG categories instead of DG algebras. In fact, Ka5 also deals with the DG category case, so that this looks like a straightforward exercise. However, since our main interest is in degeneration in char 0 , we do not go into it to save space. 
5.2 Hodge-to de Rham degeneration. We now fix a field $K$ of characteristic 0 , and a DG algebra $A$. over $K$. In this case, a Hodge-to-de Rham degeneration theorem is an immediate corollary of Theorem [2.22, and the argument is exactly the same as in [Ka2, Subsection 5.3]. We reproduce it for the sake of completeness and for the convenience of the reader.

Theorem 5.4. Assume that the DG algebra $A$. is homologically smooth and homologically proper. Then the Hodge-to-de Rham spectral sequence (2.2) degenerates, so that there exists an isomorphism $H_{\text {. }}\left(A_{\bullet}\right) \cong H H_{\bullet}\left(A_{\bullet}\right)((u))$.

We recall that homologically proper simply means that $A$. is a perfect complex over $K$ (in particular, it is homologically bounded).

Proof. By a theorem of B.Toën [T], there exists a finitely generated subring $R \subset K$ and a homologically smooth and homologically proper DG algebra $A_{\bullet}^{R}$ over $R$ such that $A_{\bullet} \cong A_{\bullet}^{R} \stackrel{\mathrm{L}}{\otimes}_{R} K$. Since $R$ is finitely generated, the residue field $k=R / \mathfrak{m}$ for any maximal ideal $\mathfrak{m} \subset R$ is a finite, hence perfect field of some characteristic $p$. Since $A_{\bullet}^{R}$ is homologically proper and homologically smooth, there is at most a finite number of non-trivial Hochschild homology groups $H H_{\bullet}\left(A_{\bullet}^{R}\right)$ and reduced Hochschild cohomology groups $\overline{H H}^{\bullet}\left(A_{\bullet}^{R}\right)$, and these groups are finitely generated $R$-modules. Then there exists a constant $N$ such that $\overline{H H}^{i}\left(A_{\text {. }}^{R}\right)=0$ for $i \geq N$. Moreover, localizing $R$ if necessary, we can further assume that $H H_{i}\left(A_{\text {. }}^{R}\right)$ is a projective finitely generated $R$-module for every $i$, and that for any maximal ideal $\mathfrak{m} \subset R, p=$ char $R / \mathfrak{m}$ is non-trivial in $\mathfrak{m} / \mathfrak{m}^{2}$ (that is, $p$ is unramified in $R$ ), and $2 p>N$. Then for any $\mathfrak{m} \subset R$ with $k=R / \mathfrak{m}$, the DG algebra $A_{\text {. }}^{k}=A_{\bullet}^{R} \stackrel{\llcorner}{\otimes_{R}} k$ satisfies the assumptions of Theorem 2.22. Therefore we have an isomorphism

$$
\overline{H P} .\left(A_{.}^{k}\right) \cong H_{\bullet}\left(A_{\bullet}^{k}\right)^{(1)}\left(\left(u^{-1}\right)\right)
$$

of finite-dimensional graded $k$-vector spaces. Since $H H_{\bullet}\left(A_{\bullet}^{k}\right)$ is concentrated in a finite range of degrees, we can replace Laurent power series in $u^{-1}$ with Laurent power series in $u$, and since $A_{\text {. }}^{R}$ is homologically smooth and proper, $A^{k}$. is also homologically smooth and proper. In particular, it is cohomologically bounded, so that Theorem 1.3 (ii) allows us to replace $\overline{H P} .\left(A_{\bullet}^{k}\right)$ with $H P .\left(A_{\text {. }}^{k}\right)$. We thus have an isomorphism

$$
H_{\bullet}\left(A_{\bullet}^{k}\right) \cong H_{\bullet}\left(A_{\bullet}^{k}\right)^{(1)}((u))
$$

of finite-dimensional graded $k$-vector spaces, so that the Hodge-to-de Rham spectral sequence (2.2) for $A_{\text {. }}^{k}$ degenerates for dimension reasons. 
Finally, since all the Hochschild homology $R$-modules $H H_{i}\left(A_{\bullet}^{R}\right)$ are finitely generated and projective, and any differential in the Hodge-to-de Rham spectral sequence for $A_{\text {. }}^{R}$ vanishes modulo any maximal ideal $\mathfrak{m} \subset R$, the differential must vanish identically. Thus the Hodge-to-de Rham spectral sequence for the DG algebra $A_{\text {. }}^{R}$ degenerates, and then so does the Hodge-to-de Rham spectral sequence for $A_{\bullet}=A_{\bullet}^{R} \stackrel{\llcorner}{\otimes}_{R} K$.

\section{References}

[Be] A. Beilinson, On the crystalline period map, Camb. J. Math. 1 (2013), 1-51, or a more recent version at arXiv:1111.3316

[BBD] A. Beilinson, J. Bernstein, and P. Deligne, Faisceaux Pervers, Astérisque 100, Soc. Math. de France, 1983.

[Bh] B. Bhatt, $p$-adic derived de Rham cohomology, arXiv:1204.6560

[C] A. Connes, Cohomologie cyclique et foncteur Ext ${ }^{n}$, Comptes Rendues Ac. Sci. Paris Sér. A-B, 296 (1983), 953-958.

[DI] P. Deligne and L. Illusie, Relévements modulo $p^{2}$ et dćomposition du complexe de de Rham, Inv. Math. 89 (1987), 247-270.

[G] A. Grothendieck, Catégories fibrée et descente, SGA I, Exposé VI, SMF, 2003.

[Ka1] D. Kaledin, Non-commutative Cartier operator and Hodge-to-de Rham degeneration, arXiv:math/0511665

[Ka2] D. Kaledin, Non-commutative Hodge-to-de Rham degeneration via the method of Deligne-Illusie, Pure Appl. Math. Q. 4 (2008), 785-875.

[Ka3] D. Kaledin, Trace functors and localization, in Contemp. Math. 643 (2015).

[Ka4] D. Kaledin, Cartier isomorphism for unital associative algebras, arXiv: 1509.08049.

[Ka5] D. Kaledin, Co-periodic cyclic homology, arXiv:1509.08784

[Ka6] D. Kaledin, Bokstein homomorphism as a universal object, arXiv:1510.06258.

[Ko1] M. Kontsevich, private communication, 2005.

[Ko2] M. Kontsevich, XI Solomon Lefschetz Memorial Lecture Series: Hodge structures in non-commutative geometry, arXiv:0801.4760

[KS] M. Kontsevich and Y. Soibelman, Notes on A-infinity algebras, A-infinity categories and non-commutative geometry, I, arXiv:math/0606241

[L] J.-L. Loday, Cyclic Homology, second ed., Springer, 1998.

[T] B. Toën, Rings of definition of smooth and proper dg-algebras, arXiv:math/0611546

Steklov Math Institute, Algebraic Geometry section AND

Center for Geometry and Physics, IBs, Pohang, Rep. of Korea

E-mail address: kaledin@mi.ras.ru 